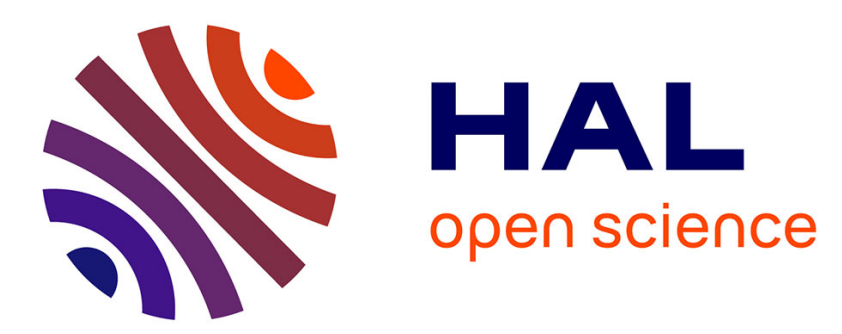

\title{
Heurs et malheurs de la légalité dans les sociétés contemporaines. Une sociologie politique de la " judiciarisation "
}

Jacques Commaille, Laurence Dumoulin

\section{To cite this version:}

Jacques Commaille, Laurence Dumoulin. Heurs et malheurs de la légalité dans les sociétés contemporaines. Une sociologie politique de la " judiciarisation ". Année Sociologique, 2009, 59 (1), pp.63-107. halshs-00433884

\section{HAL Id: halshs-00433884 \\ https://shs.hal.science/halshs-00433884}

Submitted on 20 Nov 2009

HAL is a multi-disciplinary open access archive for the deposit and dissemination of scientific research documents, whether they are published or not. The documents may come from teaching and research institutions in France or abroad, or from public or private research centers.
L'archive ouverte pluridisciplinaire HAL, est destinée au dépôt et à la diffusion de documents scientifiques de niveau recherche, publiés ou non, émanant des établissements d'enseignement et de recherche français ou étrangers, des laboratoires publics ou privés. 
$<$ TITRE > Heurs et malheurs de la légalité dans les sociétés contemporaines. Une sociologie politique de la « judiciarisation »

[Titre abrégé : Une sociologie politique de la « judiciarisation »]

Jacques Commaille et Laurence Dumoulin Institut des sciences sociales du politique - Pôle Cachan, Ecole normale supérieure

commail@isp.ens-cachan.fr, laurence.dumoulin@isp.ens-cachan.fr

Ecole normale supérieure

ISP

61, avenue du Président Wilson

F 94235 Cachan Cedex

Tél. : 0147405967 
$<$ TEXTE $>$ La littérature internationale portant sur la «judiciarisation ${ }^{1}$ accorde une importance exceptionnelle à ce phénomène qu'elle présente comme étant de très grande ampleur, touchant un grand nombre d'espaces nationaux et transnationaux, de juridictions de différents degrés. C’est en tout cas ce que révèle le travail d'inventaire que nous avons entrepris autour de cette littérature principalement anglophone. Loin de prétendre en offrir un inventaire systématique, le présent article s'appuie sur certaines des analyses, débats et controverses qui y sont développés et s'en saisit comme autant de pistes de réflexion et de nouvelles perspectives de théorisation sur les rapports entre légalité et pouvoir politique, ceci dans la mesure où cette judiciarisation, indissociable de nouveaux usages du droit dans la justice, implique également de nouveaux rôles et un nouveau statut du juridique par rapport au politique. Dans une perspective de sociologie politique du droit, l'objectif est donc bien de rendre compte de ce travail encore en cours et de mettre au jour les différents aspects et controverses qui traversent ce mouvement de judiciarisation - qu'il relève d’une réalité objectivée ou de représentations plus ou moins intentionnelles, ce que nous tenterons de préciser. Nous nous demanderons plus particulièrement dans quelle mesure cette littérature témoigne de changements ou de l'expression d’une volonté de changements dans le régime de légalité et de transformations réelles ou souhaitées des rapports entre légalité et pouvoir politique. En effet, la question fondamentale que pose le développement d'une littérature sur la judiciarisation est de trois ordres : 1- Constitue-t-il une des expressions d'une évolution des

\footnotetext{
${ }^{1}$ A ce stade, plusieurs centaines de références ont été répertoriées - ouvrages et articles confondus - dans leur l'immense majorité en langue anglaise. Le présent article repose sur un dépouillement d'une partie de cette littérature et ne saurait par conséquent prétendre constituer un inventaire exhaustif. De plus, il nous faut assumer le fait que nous ne sommes pas encore en mesure de situer cette littérature sur une échelle chronologique. Rapporter les analyses produites à leurs périodes de production offrirait certainement un facteur explicatif supplémentaire et permettrait de mieux saisir le sens de cette mobilisation sur le phénomène de judiciarisation. Nous ne pouvons malheureusement solliciter cette dimension explicative à ce jour.

Par ailleurs, nous avons déjà consacré une première analyse à ce phénomène : Jacques Commaille et Laurence Dumoulin, «Droit, justice et politique : pour une lecture critique de la littérature », Congrès de l'Association Québécoise de science politique, Université Laval, Québec, 25-26 mai 2007. Les auteurs tiennent à remercier les organisateurs de l'atelier consacré aux « Causes, formes et conséquences de la judiciarisation », qui leur ont permis de tester ainsi leurs premiers résultats, en particulier Christine Rothmayr, laquelle les a fait bénéficier de son excellente connaissance de la littérature internationale sur ce thème et de ses remarques judicieuses.
} 
formes de la légalité signifiant une transformation du principe de légitimité de la domination politique ? 2- Représente-t-il une des manifestations de la remise en cause du monopole de l'Etat moderne à produire le droit ? 3- Annonce-t-il une transformation des modes de gouvernement?

Mais avant de rechercher le sens qui peut être donné à cette mobilisation de la littérature savante sur la question de la judiciarisation, il convient de tenter de définir ce qui est entendu par ce terme et de cerner la réalité qu’il est censé recouvrir (1). Ce travail préalable de définition devrait permettre ensuite de souligner quelques-unes des difficultés d'un positionnement de sciences sociales sur un objet aussi incertain par rapport aux faits qu'il est supposé qualifier, aux variations dont il est l'objet en fonction de contextes institutionnels, politiques, et culturels différents (2). Parmi les nombreuses questions abordées par cette littérature internationale, deux axes thématiques seront privilégiés dans le cadre de cet article : la mise en exergue du rôle accru et de plus en plus crucial de la justice dans la production de politiques publiques (3) laquelle est complémentaire d'un second aspect concernant la mise en lumière du caractère proprement central de la justice dans le fonctionnement démocratique (4). En effet, ces deux approches nous paraissent susceptibles de poursuivre au plus près l’objectif qui est le nôtre, à savoir contribuer à la compréhension de la place traditionnellement accordée à la légalité et au rôle que la justice tiendrait désormais dans la constitution du politique et dans son fonctionnement. Ce qu'est susceptible de révéler cette littérature, à travers les controverses qui habitent la façon dont ces deux thèmes sont traités (dont nous ne dissimulerons rien de leur caractère souvent contradictoire), c’est bien un changement du statut et de régime de la légalité, en rapport avec les transformations du politique et d'où découlerait le constat - ou l'affirmation incantatoire - d'une « judiciarisation ». 
Dans cette perspective, la question n’est pas seulement de savoir si la judiciarisation est une réalité objectivée ${ }^{2}$. Si elle est aussi plus de l'ordre de l'intention ou de l'expression d'une volonté, cela peut signifier qu'elle est imposée comme une représentation dans le but de perpétuer une conception de la légalité non seulement comme fondement du pouvoir mais aussi comme pouvoir. Ainsi, loin d'apparaître comme un phénomène qui serait propre à la justice, qui relèverait exclusivement d'une sociologie de la justice, la judiciarisation et les façons dont elle est définie, analysée, promue dans la littérature internationale, constitueraient bien pour nous un révélateur privilégié de la place occupée par la légalité, ou que certains auteurs et/ou acteurs sociaux aspirent désormais à lui faire occuper dans la régulation politique.

\section{<IT1>1- La « judiciarisation-judicialization », un phénomène polymorphe}

Sous le terme de «judiciarisation» («judicialization») coexistent des significations nombreuses et variées. Comme le souligne un auteur: «la judiciarisation est une manifestation importante de la vie politique contemporaine (...) mais elle découle d'une diversité de causes, prend des formes différentes, et peut aboutir à des résultats tout à fait hétérogènes d'un système politique à un autre » (Waltman, 1996, p. 685). Il demeure que pour de nombreux auteurs, il est question d'expansion du pouvoir judiciaire pour reprendre le titre d'un ouvrage de référence en la matière (Tate et Vallinder, 1995) ou encore d' « accroissement du pouvoir judiciaire » (Guarnieri et Pederzoli, 2002) signifiant la montée en puissance ou même la prise de pouvoir des acteurs judiciaires. En référence à l’objectif théorique que nous poursuivons ici, nous accorderons alors une particulière importance à la définition suivant

\footnotetext{
${ }^{2}$ De façon générale, nous mesurons toute la difficulté d’appréhender le réel sur cet objet qu'est la justice dans la mesure où, plus que pour d'autres institutions, la relative rareté de données de sciences sociales sur ce qu'elle fait et ce qu'elle produit socialement et politiquement contraste avec l'abondance des références dont elle fait l'objet dans le débat social et politique, notamment à travers ce qu'en rapportent les médias (sur le rôle déformant des medias, voir en particulier : Haltom et McCann, 2004).
} 
laquelle la judiciarisation fait référence à « un déplacement de grande ampleur du pouvoir, s’observant au niveau international, du Législatif vers le judiciaire et les autres institutions juridiques » (Ferejohn, 2002). Les usages des termes de «jurocracy» (Horowitz, 1977), de «juristocracy» (Hirschl, 2004) ou de «courtocracy» (Scheppele, 2002) renvoient à cette même acception : celle d'un système politique où les acteurs dominants du jeu politique deviennent les professionnels de la justice, où « le pouvoir décisionnel se déplace devant les tribunaux » (Fournier et Woehrling, 2000, p. 4). En particulier, les juges seraient davantage associés à la vie politique et à l'action publique selon une triple dimension : dans l'imposition de limites substantielles au pouvoir des institutions législatives, dans la définition du contenu même des politiques publiques et de leur mise en œuvre concrète et enfin dans l'arbitrage de l'activité politique elle-même via la régulation de la compétition politique - à travers le financement des partis ou encore le traitement du contentieux électoral (Ferejohn, 2002).

Cette notion de «judiciarisation » ferait particulièrement sens dans des systèmes démocratiques, lorsqu'il existe une forme de séparation des pouvoirs à la Montesquieu et des politiques de protection des droits individuels, notamment des droits des minorités - y compris lorsqu'ils vont à l'encontre de ceux de la majorité. La faiblesse et l'inefficacité 'objectives' des institutions majoritaires - comme les partis politiques et les coalitions gouvernementales lorsqu'ils ne parviennent pas à s'organiser - au même titre que la perception négative par les élites et l'opinion publique des institutions qui participent au processus de production de l'action publique seraient autant de facteurs contribuant à faire des acteurs judiciaires des acteurs de plus en plus stratégiques du système politique. Parallèlement ou cumulativement, les stratégies contentieuses déployées par de tiers acteurs comme les groupes d’intérêt ou les partis de l’opposition pourraient également avoir pour effet de mettre les acteurs judiciaires au centre du jeu, de même que la tendance de certains 
acteurs politiques à ne pas traiter des problèmes dont ils sont pourtant saisis (Tate et Vallinder, 1994).

Pour certains, la judiciarisation renvoie au processus général par lequel « le discours du droit - normes de comportement et langage - pénètre et est absorbé par le discours politique » (Shapiro et Stone Sweet, 2002, p. 187). Ainsi « des «politiques judiciarisées » sont des politiques poursuivies finalement en partie à travers la médiation du discours du droit » (Shapiro et Stone Sweet, 2002, p. 187). Ceci peut alors amener à considérer que « le discours politique est maintenant imprégné du langage du droit et de la légitimité ». De même « qu’en matière de realpolitik, les juristes sont aussi centraux que le sont les stratèges dans une campagne militaire, [...] les droits sont autant une ressource de pouvoir que les armes ou l'argent, et la souveraineté juridique, fondée sur les normes juridiques de la société internationale, devient une clef déterminante du pouvoir d’Etat » (Reus-Smit, 2004, p. 2).

Mais la notion de judiciarisation recouvre également le développement de procédures de type judiciaire dans des forums non judiciaires de prise de décision et de négociation (administrations nationales ou internationales comme le GATT...) (Tate et Vallinder, 1994). Par cet usage du terme, il serait plutôt fait référence à une sorte de mimétisme institutionnel qui expliquerait que les méthodes judiciaires seraient importées et adaptées dans d’autres secteurs. La forme du procès réglé par un tiers impartial, le principe du contradictoire, la possibilité de faire appel, l’obligation de motiver toute décision seraient autant de dispositions empruntées au modèle judiciaire qui se diffuseraient au sein d'autres administrations, notamment par la circulation d'acteurs entre ces différents espaces. Il s'agirait là d'une judiciarisation de l’intérieur («from within ») par opposition à la précédente qualifiée de judiciarisation de l'extérieur («from without») (Vallinder, 1994, p.92-93). Cette forme de judiciarisation marquée par des recours aux procédures para-judiciaires provoquerait une 
surprocéduralisation dans les processus de production des politiques publiques américaines, ce qui est dénoncé dans des réflexions de nature doctrinale (Smith, 1985; McGowan, 1986). L’importance accordée à la justice «restauratrice » dans des situations comme celle des violences de l'apartheid en Afrique du sud, de la dictature chilienne ou des dictatures dans d'autres pays d'Amérique Latine, représente une autre forme d'expression de ce phénomène de judiciarisation (Hirschl, 2002 ; Sugarman, 2002 ; Lefranc, 2007) comme, de façon plus générale, la multiplication des juridictions supranationales (une vingtaine auraient été créées depuis 1946 : Alter, 2006).

Si le cas américain est très souvent évoqué dans cette littérature, le phénomène de la judiciarisation est considéré d'emblée comme ayant un caractère mondial. S’agissant plus spécifiquement de son expansion en Europe, quelques causes principales sont généralement avancées : une multiplication des niveaux de gouvernement et d'administration notamment dans le cadre d'un processus de fédéralisation ou de constitution d'entités politiques supranationales (comme l’Union Européenne ${ }^{3}$ ), la perte de confiance dans les gouvernements « technocratiques », la démocratisation et l’établissement de sphères privées de la vie sociale garanties par un renforcement judiciaire des droits, l'expansion des groupes de pression et l'implication croissante des citoyens dans la réalisation des politiques publiques (Shapiro et Stone, 1994, p. 402). A ces causes, il conviendrait certainement d'ajouter l'institution de nouvelles justices dans les pays d’Europe ex-communistes (Coman et De Waele, 2007).

De façon générale, la judiciarisation serait une des manifestations d'une "globalisation judiciaire » concomitante des «globalisation[s] juridique » et économique. Cette « globalisation judiciaire », constitutive d’une «communauté de juridictions », prendrait notamment la forme de coordinations de plus en plus fréquentes («judicial comity») entre

\footnotetext{
${ }^{3}$ Dans plusieurs travaux, la création de l’Union Européenne est présentée comme contribuant fortement au développement du phénomène de judiciarisation (voir notamment : Stone Sweet, Sandholtz et Fligstein, 2001). Elle aurait ainsi, par exemple, favorisé l'importation de l' « adversarial legalism » américain (Kelemen, 2006) et fait de la Cour Européenne de Justice la juridiction internationale « la plus influente au monde » (Alter, 1996. Sur l'influence des juridictions européennes, voir également : Alter, 1994 ; 1998 ; Volcansek, 1997).
} 
juridictions et juges de différents pays et de «fertilisations croisées des cultures juridiques » (Slaughter, 2000 ; 2003).

Il convient de souligner que ces expressions de la judiciarisation apparaissent, de façon très majoritaire, dans des univers judiciaires circonscrits. C'est la justice administrative et constitutionnelle (celle-ci dans le cadre du «new constitutionalism ») qui est largement privilégiée dans l’observation du phénomène et non pas la justice judiciaire (Koopmans, 2003 ; Hirschl, 2002 ; 2004 ; Ginsburg, 2003 ; Revue internationale de science politique, 1994 ; Tate et Vallinder, 1994). Ce qui explique, par exemple, que la principale forme de judiciarisation puisse être le judicial review c'est-à-dire le contrôle des lois par les tribunaux (Vallinder, 1994 ; Hertog et Halliday, 2004) ou, plus précisément encore, le « constitutional review » (Shapiro et Stone, 2004). Une telle perspective n’est pas surprenante dans la tradition américaine où le rôle de la Cour suprême est depuis longtemps au centre des préoccupations ainsi qu'en témoigne par exemple une analyse ancienne de Robert Dahl encore discutée aujourd'hui (Dahl, 1957). Mais ce qui est désormais en question, c’est à la fois l'extension internationale du phénomène et la nature même du rôle de la Cour Suprême, la façon dont elle s'inscrit dans le jeu politique institutionnel global et les analyses qui peuvent en être données (Sunstein et Epstein, 2001 ; Epstein, Knight et Martin, 2001). Sur ce point, la mise en place de cours constitutionnelles dans les pays d'Europe ex-communistes est considérée comme une manifestation significative du phénomène (voir, par exemple, Solomon Jr et Foglesong, 2000 ; Epstein, Knight et Shvetsova, 2001 ; Maveety et Grosskopf, 2004). Il en est de même pour ce qui concerne la Cour Suprême en Israël (Edelman, 1994 ; Dor et Hofnung, 2006 ; Mizrahi et Meydani, 2003), en Allemagne, en Amérique latine ou dans les pays asiatiques (Ginsburg, 2003), l’objectif étant notamment de valoriser ou de discuter la capacité réelle des tribunaux à produire du changement social, et ce, à partir de 
décisions considérées comme marquantes de ces Cours Suprêmes (Rosenberg, 1991 ; Canon et Johnson, 1998).

Enfin, le phénomène de judiciarisation («judicialization ») est souvent mis en relation avec un processus plus global d'expansion et de mutation de la légalité, celui de la « juridicisation » («juridicalization») (Wright, 1999; Commaille et al., 1999 ; Shapiro, 1994). La judiciarisation serait ainsi globalement une forme de juridicisation mais il resterait à préciser la nature de la relation, plus complexe et moins univoque qu'il n’y paraît au premier abord, entre les deux phénomènes. Par exemple, le cas de la Suède tend à laisser penser que la judiciarisation ne serait pas dépendante de la juridicisation mais qu'elle se déploierait, au contraire, dans un contexte de reflux de la place des juristes dans la vie politique. Ce serait parce que les professionnels du droit seraient vus comme moins présents, moins influents dans l'appareil d'Etat (essentiellement le Parlement et le Gouvernement) que les tribunaux seraient davantage sollicités et renforcés comme un troisième pouvoir. Il y aurait eu d'un côté 'dejuridicalization' au sens de perte d'importance des juristes dans la vie politique et l'appareil étatique suédois et d’un autre côté 'judicialization', au sens où cela aurait provoqué une propension accrue à avoir recours à la branche judiciaire proprement dite et à attendre d'elle un rôle plus actif, notamment dans le contrôle des décisions gouvernementales puis, à partir de 1974, dans le contrôle de constitutionnalité des lois (Holmström, 1995). La judiciarisation ne peut donc être résumée à une forme de juridicisation des rapports sociaux, comme on pourrait être tenté de le penser à la lecture de certains auteurs. Suivant les configurations historiques et nationales, il est probable que les phénomènes s’articulent de façons différenciées.

\section{$<$ IT1 $>$ 2- La judiciarisation et les pièges de l’objet en sciences sociales}


$<$ IT2> 2.1- Les implicites

La littérature qui mobilise la notion de judiciarisation repose sur un certain nombre d’implicites qu'il convient précisément d'expliciter dans la mesure où ils révèlent quelques pré-requis sous-jacents. Bien entendu, il ne s’agit pas de disqualifier notre objet mais de ne pas ignorer les constructions auxquelles il est exposé, constructions constitutives et par conséquent révélatrices de son statut spécifique par rapport au politique.

C’est d'abord le caractère linéaire du changement qui sous-tend le processus de judiciarisation lui-même et qui, de fait, caractérise une grande partie des analyses qui lui sont consacrées. Un processus massif, unilatéral dont la signification politique serait univoque (Roussel, 2003) est ainsi privilégié. Il s’agit d’admettre un changement d'un état A vers un état B dont le résultat est finalement assez simple: le déclin de la souveraineté parlementaire au profit d'une légitimité judiciaire, le transfert de pouvoir des acteurs politiques vers les acteurs judiciaires. Les travaux qui se revendiquent le plus explicitement comme des tentatives de constituer théoriquement la notion de judiciarisation se reconnaissent d'abord dans une définition qui associe certes l'idée d'un accroissement du recours aux juridictions et aux professionnels du droit mais promeuvent surtout l'idée d'une accentuation de la fonction politique de ces instances dans le système politique global (Tate et Vallinder, 1994). En ce sens, ils s’inscrivent dans le sillage de Martin Shapiro et Alec Stone lesquels analysent plus spécifiquement l'émergence d’une tendance globale de « constitutionnalisation du politique » (Shapiro et Stone, 1994). Nombre d'autres auteurs vont soutenir ces mêmes thèses (par exemple Ginsburg, 2003 ; Guarnieri et Pederzoli, 2002).

Torbjörn Vallinder tente de distinguer deux formes idéales-typiques de la protection des droits dans les démocraties : protection des droits et obligations de la majorité par le pouvoir législatif d'un côté versus protection des droits individuels par les tribunaux de l'autre. Pour lui, l’idée de judiciarisation recouvre le passage de la première vers la seconde forme de 
protection des droits, les acteurs gagnants de cette mutation étant les professionnels de la justice et du droit alors que les perdants en seraient les acteurs de la classe politique (Vallinder, 1994). Dans toutes ces analyses, l'idée d'un changement, d'une transformation des modes de construction du politique est privilégiée, en particulier sous la forme d'une tendance repérable de « judiciarisation des politiques publiques » dont la France de la Ve République est parfois choisie comme cas exemplaire (Wright, 1999, p.97).

Outre de servir à la description d'un phénomène linéaire, empreint d’un certain déterminisme historique, la notion de judiciarisation impose la représentation d'un processus pensé comme global et universel, touchant tous les systèmes politiques et juridiques à travers le monde. Là encore, c’est explicitement cette direction qu'empruntent Neal Tate et Torbjörn Vallinder quand ils évoquent ce qui serait un processus à l'œuvre dans « tous les systèmes politiques dans le monde » (Tate et Vallinder, 1995, p.27), autrement dit « a world wide phenomenon » (Vallinder, 1994, p.91). Or, cette approche n'est pas sans effet sur la nature des projets intellectuels et des systèmes explicatifs qui sont déployés dans cette perspective. En effet, elle conduit ces auteurs à se donner pour ambition et question de recherche légitime l'établissement d'un état des lieux de la judiciarisation à travers toute une série de pays. Ce panorama leur permet ensuite, à partir des éléments observés dans les différentes configurations nationales, de recenser les conditions favorables au développement de la judiciarisation. Différents phénomènes sont alors traités comme des signes, des indices d'un même processus, saisissable et reconnaissable à travers des situations hétérogènes. La diversité des types de juridictions et d'acteurs concernés, des espaces nationaux ou internationaux observés, des systèmes juridiques et politiques considérés n’est pas pensée comme rédhibitoire à ce type d'entreprise. Elle est au contraire thématisée sous l'angle de la diversité des formes que prend la judiciarisation à travers le monde. Il reste que la démarche produit aussi un effet d'uniformisation puisque sont mis sur le même plan des phénomènes 
qui relèvent de réalités sociales non connectées entre elles. Tim Koopmans passe par exemple du cas de l'extension du contrôle de constitutionnalité des lois dans l'Europe continentale, à celui du rôle des tribunaux américains sur des questions sensibles comme l'avortement et à celui de l'engagement judiciaire dans les problèmes européens et internationaux (Koopmans, 2003), sans forcément poser la question des éventuelles circulations entre ces différentes scènes judiciaires. Un autre auteur quant à lui traite ensemble trois pays asiatiques (Taïwan, la Chine et la Mongolie) pour expliquer la mise en place d'un mécanisme de contrôle des lois (judicial review) qu'il interprète comme une forme de judiciarisation propice à la montée en puissance des acteurs juridictionnels (Ginsburg, 2003). Les trois pays, placés sur le même plan, sont considérés comme des exemples du déclin avéré de la souveraineté parlementaire au profit du contrôle de constitutionnalité opéré par des instances judiciaires, dans le droit fil de l'exemple américain notamment (Ginsburg, 2003). Un sens commun est ainsi conféré au phénomène de judiciarisation, lequel est étroitement associé aux processus de démocratisation et interprété comme synonyme d’un progrès dans la protection des droits individuels.

Le travail de généralisation ainsi opéré susciterait moins de réserve si la judiciarisation était notamment rapportée aux contextes inspirés par des traditions juridico-politiques différentes. Faut-il rappeler le poids qu'il convient d'accorder aux traditions de la common law d'une part, à celles du droit romano-germanique d'autre part ? Dans le premier cas, le droit est d'abord associé à la société, il est un droit immanent, un law in action, susceptible d'être, comme le souligne Talcott Parsons lui-même, un droit des individus, des collectivités privées contre l'arbitraire du pouvoir, de l'intervention gouvernementale (Parsons, 1962). Dans le second cas, le droit est l'expression de la souveraineté de l'Etat, un droit référence, un law in books. Pour ne prendre qu'un exemple concernant ces différences entre systèmes de droit et les rapports qu'ils induisent entre justice et politique, Martin Shapiro et Alec Stone Sweet remarquent que le phénomène du «constitutional judicial review » au cours du demi siècle 
précédent a fait apparaître deux modèles dominants de « review » : un modèle européen et un modèle américain. En Europe, les générations successives d'hommes politiques, les rédacteurs de constitutions et les legal scholars ont eu le souci de rejeter le modèle américain de judicial review donnant un pouvoir de contrôle du judiciaire sur le politique pour privilégier des variations autour d’un système que Hans Kelsen considérait comme adapté aux institutions parlementaires, à l’idéologie de la séparation des pouvoirs et à celle de la légalité d'une review constitutionnelle mais non judiciaire. Dans cette conception, qui sera appliquée à la Cour Constitutionnelle autrichienne de la seconde République (1920-1934) puis inspirera les modèles européens, la suprématie du législatif implique la séparation des pouvoirs ; de celle-ci découle la subordination de la fonction judiciaire à la fonction politique (Shapiro et Sweet, 2002, p. 343). Si dans le système américain, «n’importe quel juge de n’importe quel tribunal, quel que soit le cas, à n’importe quel moment, à la requête de n’importe quel justiciable, a le pouvoir de déclarer une loi anticonstitutionnelle » (Stone Sweet, 2000, p. 32), il n'en va pas de même dans les systèmes continentaux. Au contraire, la subordination du travail du judiciaire à celui du législatif est un principe fondamental des systèmes de droit civil et, par conséquent, du droit constitutionnel continental. La référence en la matière est le principe édicté en 1790 en France : «Les tribunaux ne peuvent pas interférer avec l’exercice des pouvoirs législatifs ou suspendre l'application de la loi » (Stone Sweet, 2000, p. 33). «Cette orthodoxie constitutionnelle s’est répandue à travers l'Europe au cours du XIXème siècle. Suivant cette orthodoxie, l'American-style judicial review, plutôt que de correspondre à une séparation des pouvoirs, établit une confusion permanente des pouvoirs. [Or,] la crainte de voir se constituer un gouvernement des juges est au fondement de l’hostilité des Européens à l’American judicial review » (Stone Sweet, 2000, p. 33).

C’est au prisme de ces diversités culturelles et de ces traditions juridiques qu'il conviendrait certainement de réexaminer ce phénomène de judiciarisation et d'admettre la très grande 
disparité possible des mécanismes regroupés sous ce terme. La tentation de l’universalité ou de l'unicité mérite aussi d'être discutée quand on considère le processus de judiciarisation d'une seule et même question. Ainsi en va-t-il par exemple de la procréation médicalement assistée. Une étude comparée portant sur le Canada, les Etats-Unis et la Suisse montre qu’en la matière, la fréquence des litiges et l’impact des décisions de justice sur les politiques publiques sont très variables et pas aussi monolithiques que la notion de judiciarisation pourrait le laisser penser (Rothmayr et L’Espérance, 2007). Les différences entre pays sont non seulement importantes mais inattendues : ce n’est pas aux Etats-Unis que les décisions de justice ont eu le plus grand impact sur les politiques publiques et ce ne sont pas non plus les caractéristiques institutionnelles des systèmes juridiques et politiques considérés qui s’avèrent ici déterminantes. Pour les auteurs, les différences sont plutôt à attribuer «aux variations des processus politiques, de la mobilisation des acteurs et du contenu des politiques publiques » (Rothmayr et L’Espérance, 2007, p.205) ${ }^{4}$.

\section{$<$ IT2 $>$ 2.2- Les locuteurs}

Les incertitudes concernant le statut scientifique des analyses consacrées à la judiciarisation, le caractère parfois prescriptif et évaluatif de ces analyses, suggèrent en outre de prêter attention aux conditions dans lesquelles elles sont produites. Cette orientation de la réflexion serait d'autant plus nécessaire que le rôle des professionnels du droit et de la justice dans la production du phénomène de la judiciarisation serait lui-même central, à la mesure du rôle qu'ils joueraient dans le cadre d'une arène juridique devenue un élément pivot dans la structuration du pouvoir politique et l'avènement du libéralisme politique (Halliday, Karpik et Feeley, 2007). Un auteur comme Martin Shapiro souligne ainsi, au fil de ses différents travaux, le rôle des juristes et notamment des cabinets d'avocats dans les campagnes judiciaires (litigation) que mènent certains groupes d'intérêt: «The lawyers created the

\footnotetext{
4 Pour une complexification des modèles d'analyse, à partir de l'exemple du rôle du judiciaire dans tout ce qui concerne la langue officielle des minorités dans l’éducation au Canada, voir également : Riddell, 2004.
} 
clients as often as the clients created the lawyers » (Shapiro, 1994, p.109). D’autres auteurs insistent également sur les facteurs endogènes au milieu judiciaire, en particulier sur la structure du système judiciaire et son caractère plus ou moins fragmenté, sur le type de formation et de recrutement des juges en vigueur, sur le comportement individuel et collectif (via les syndicats et associations professionnelles) des juges eux-mêmes, tout ces facteurs étant susceptibles de peser sur le phénomène de judiciarisation (Guarnieri et Pederzoli, 2002). Cela explique certainement que soit parfois souligné le caractère typiquement indigène, interne à la sphère juridique de bien des analyses qui portent sur le droit et la justice, sur leur rapport avec le politique et sur la notion de judiciarisation. C’est la raison pour laquelle un auteur comme Martin Shapiro impose l’idée d'une «political jurisprudence » en considérant que cette notion ainsi que le positionnement qu'elle implique représente à la fois le prolongement et une forme de dépassement de certains éléments internes à la production de connaissance sur le juridique comme la sociological jurisprudence ou le réalisme judiciaire. Le recours aux connaissances fondamentales de la science politique est de nature, selon Shapiro et Sweet, à alimenter et ancrer ce positionnement (Shapiro et Sweet, 2002, p. 19). Une telle position de rupture par rapport à une vision internaliste de l'approche du juridique est certes à resituer dans le contexte américain où les analystes s’accordent depuis longtemps à souligner la place privilégiée de la justice dans le système américain de gouvernement et la dimension politique du droit et de la justice.

Pour Martin Shapiro, le système américain est traditionnellement appuyé sur le droit : la révolution américaine a été pensée comme la revendication de droits juridiques et constitutionnels d’individus à l'égard du Parlement, c'est-à-dire qu'elle a été pensée avant tout sur un mode juridique (Shapiro, 1994). Toute une série d'autres raisons qui tiennent à la structuration du système politique américain (structuration d'un bipartisme dans le rapport à la Constitution, rôle de la Cour suprême dans l'arbitrage des relations entre un Etat fédéral et 
des Etats fédérés et dans la construction d'un gouvernement central fort...) se conjuguent pour faire de la forte présence des juristes dans la vie américaine une caractéristique majeure de ce système. En fait, depuis les premiers jours de la République, les tribunaux ont acquis des pouvoirs dont on ne trouve pas d'équivalents dans d'autres pays. La référence à Tocqueville (De la démocratie en Amérique) s’impose alors puisque celui-ci souligne la place importante du légalisme dans la culture américaine et la propension des Américains à penser les problèmes sociaux en termes légaux (Horowitz, 1977). Le juge est une figure politique centrale du champ juridique et les juridictions y sont en effet reconnues comme des « institutions politiques » (Smith, 1997). Il est banal de considérer que les affaires de justice y sont perçues comme des affaires politiques, que le rapport du judiciaire au politique est particulièrement fort (Jacob, et al, 1996). Les tribunaux ne sont pas « des institutions légales formelles qui sont influencées par un environnement politique, mais sont considérés comme un élément majeur faisant partie intégrante de la politique des Etats et de la politique nationale » (Glick, 1983, p. X). Dans ce cadre, le statut des juges locaux est volontiers associé au destin de la communauté (Auerbach, 1983). Le juge est « connecté à la société » (Smith, 1997) ou plus encore à la réalisation d’une " popular democracy» (Champagne \& Haydel, 1993), la fonction de justice étant plus généralement considérée comme un « élément central du projet démocratique » (Cannon \& O’Brien, 1985). Ces analyses soulignent ainsi que cette dimension politique de la justice ne saurait valoir seulement pour la Cour Suprême ou les Cours supérieures. Elle concerne également les tribunaux locaux dont on suppose le rôle important qu'ils jouent dans le processus de production des politiques des gouvernements locaux (Shapiro et Stone Sweet, 2002, p. 36). L’évidence s’impose alors : l'étude de la justice comme du droit relève d'abord de la science politique (sur cet aspect de la littérature américaine sur la justice, voir : Commaille, 2000). 
Martin Shapiro et Alec Stone Sweet érigent donc en objet de recherche l'affirmation constante que font les juristes de leur indépendance, de leur neutralité, de leur apolitisme. Ils considèrent que la mise en avant de ces attributs n'est autre qu'un moyen de renforcer leur légitimité auprès du politique. Dans cet esprit, il s’agit de rompre avec ce qui serait la tendance des juristes à revendiquer un monopole dans le travail d'observation et de commentaire sur le droit et la justice. Pour ces auteurs, ce parti-pris de rupture est d'ailleurs d'autant plus difficile à mettre en œuvre que les juristes ont pour eux une propension toute particulière à jouer subtilement de la confusion des registres entre la pratique professionnelle, le travail de connaissance, la poursuite d’intérêts et la défense de valeurs. Rien ne le démontrerait mieux que leur aptitude à s’insérer dans des communautés épistémiques et à tirer avantage d'espaces intermédiaires entre l'échange de connaissances, l'expertise et la contribution à l'exercice du pouvoir (Shapiro et Stone Sweet, 2002). C'est ce qui explique que ces juristes puissent être considérés comme une "policy community » agissant sur les autres communautés politiques à travers notamment son activité doctrinale, laquelle est un instrument sophistiqué d'exercice d'un pouvoir d'influence sur le politique (Shapiro et Stone, 2002). On comprend alors que Shapiro et Sweet estiment que « les professeurs de droit constitutionnel ont fait plus qu'aucun autre groupe appartenant aux élites de la société européenne pour favoriser et légitimer le développement de la constitutional review et, par voie de conséquence, contribué à la promotion de l’idée de judiciarisation » (Shapiro et Stone Sweet, 2002, p. 415). Derrière le phénomène de judiciarisation, on pourrait ainsi distinguer le rôle d'acteurs juristes, ce qui rendrait d'autant plus impérieuse l'autonomie du regard de sciences sociales sur l'objet. Martin Shapiro et Alec Stone Sweet s'autorisent à rappeler que les lawyers scholars sont encore des lawyers et que la constitution d'une science sociale autonome du droit et de la justice est d'autant plus nécessaire que « le droit doit être compris non pas comme un phénomène spécifique mais comme partie intégrante du système social » 
(Shapiro et Stone Sweet, 2002, p. 19). Pour ces auteurs, la pratique du droit est d'abord une conduite humaine avant d'être du droit et, par conséquent, elle exige d'être étudiée par des chercheurs de sciences sociales et non par des juristes. C'est en respectant cette exigence que, selon Lee Epstein et Jack Knight cette fois, les travaux sur les tribunaux et les juges aux EtatsUnis sont passés de simples analyses doctrinales à de véritables travaux de recherche où la théorie « guide une recherche empirique » (Epstein et Knight, 2004, p. 174).

Pour Gerald Rosenberg, l'affirmation de ce positionnement de sciences sociales est d'autant plus nécessaire que les juristes n'accordant pas d'attention aux preuves empiriques d'une part et à la littérature de sciences sociales d'autre part, « une grande partie de la littérature sur les juridictions est fondamentalement imparfaite » (Rosenberg, 1991, p. 342). Il s’attache alors à fournir lui-même une démonstration de ce positionnement de recherche autonome par rapport à la perspective «juridiste» (Rosenberg, 1991). Il adopte pour cela une perspective d'objectivation et de systématisation de ses analyses qui tranche avec bien des approches classiques des questions des rapports entre justice et autres acteurs dans le changement social. Il confronte les arguments des uns et des autres aux sources qui peuvent empiriquement permettre de les vérifier. Il se réclame d’une approche qui se distingue des juristes de droit constitutionnel (constitutional scholars), lesquels produisent des théories mais sans jamais regarder ce qu'il en est en pratique, dans la réalité de la Cour Suprême et des contraintes qui se posent à elle.

Outre la vigilance qu'elles suggèrent à l'égard des constats avancés dans la littérature sur la judiciarisation, ces considérations critiques permettent de comprendre les bilans contrastés que cette littérature révèle pour les deux axes thématiques que nous avons choisi d'évoquer dans cet article, à savoir le rôle de la justice dans la fabrique des politiques publiques d’une part et dans le fonctionnement démocratique d'autre part. 


\section{$<$ IT1 $>$ 3- Les mises en ouvre de la légalité comme politiques publiques}

Un des aspects majeurs de la judiciarisation consiste à considérer que les tribunaux sont devenus des institutions qui comptent dans la définition du contenu même et de la fabrique des politiques publiques (Ferejohn, 2002), notamment aux Etats-Unis. Cette transformation interviendrait dans le cadre d'un développement du judiciaire qui s’effectuerait aux dépens de l'administration et des acteurs politiques, donc dans le cadre d'un transfert du processus de decision making des droits du Parlement, du gouvernement et de l'administration vers les tribunaux (Ran Hirschl emploie ainsi l'expression de «judge-made policy-making », Hirschl, 2002). La justice fédérale et la Cour Suprême américaine sont dès lors décrites comme de véritables lieux de production des politiques publiques. Comme l’affirment deux auteurs : « le judiciaire structure ou donne les orientations nécessaires à la politique publique » (Cannon \& O’Brien, 1985, p. 253), par exemple en ce qu’il précise les conditions d'application de tel ou tel texte et contribue à fixer le cadre de telle ou telle politique publique à conduire. Ainsi, la place de plus en plus importante occupée par le judiciaire dans la régulation politique (sur laquelle nous nous pencherons infra) est indissociable du rôle joué dans ce domaine des politiques publiques, de telle sorte que pour Martin Shapiro par exemple, l'analyse comparative qu'il entreprend sur l'Angleterre, l'Europe occidentale, la Chine impériale et au sein de l’Islam, le conduit à considérer que le rôle des tribunaux dans le renforcement de la légitimité du régime politique peut s’accompagner d'une intervention de la justice dans «l'allocation de ressources économiques ou de l'établissement de véritables politiques sociales » (Shapiro et Stone Sweet, 2002, p. 63).

$<$ IT2> 3.1- Les tribunaux comme acteurs importants des politiques publiques

Dès la fin des années 1970, Donald Horowitz observe que depuis une ou deux décennies la société américaine se tourne de plus en plus vers les tribunaux pour résoudre certains de ses 
problèmes les plus complexes, par exemple les questions d'intégration, le contrôle de la police, la protection de l'environnement ou l'amélioration du sort des pauvres (Horowitz, 1977). Gerald Rosenberg, qui écrit vingt ans plus tard, cite non seulement Donald Horowitz mais aussi toute une série d'autres auteurs qui, depuis les années 1980, défendent l’idée selon laquelle les tribunaux américains et en particulier la Cour Suprême seraient devenus les principaux artisans du changement social dans certains domaines comme celui des droits civils, des droits des femmes ou encore du droit pénal et des prisons (Rosenberg, 1991). Des procès et décisions judiciaires marquants - comme la décision Brown v. Board of Education de la Cour Suprême des Etats-Unis qui interdit la ségrégation raciale dans les écoles publiques au milieu des années 1950 - auraient donné de nouvelles directions à l'action publique, d'une part parce que seuls les tribunaux, n’étant pas soumis aux contraintes électorales, auraient été en mesure de les impulser, d'autre part parce qu'une fois les premières décisions prises, ils auraient joué un effet d’entraînement sur les autres pouvoirs et sur l’opinion publique.

Le processus de policy-making serait donc passé d’un triangle de fer (les comités du Congrès, les groupes d'intérêt, la ou les agences administratives compétentes) à un rectangle de fer intégrant désormais un nouvel acteur, en l’occurrence la Cour Suprême et les Cours d’Appel fédérales, lesquelles ont généré tout un corpus de nouvelles règles juridiques visant à assurer le contrôle sourcilleux des actes administratifs (Shapiro, 1994).

Au fil du temps, la justice fédérale américaine et en particulier la Cour Suprême a ainsi développé une politique de lutte contre les inégalités (raciales, sexuelles...) ou encore une politique de régulation du fonctionnement des prisons (Feeley et Rubin, 1998). Le rôle de la Cour Suprême en la matière se mesure notamment à sa capacité à maîtriser son agenda en procédant à des choix d'affaires à traiter en fonction de sa propre perception de l'importance politique de celles-ci (McGuire et Palmer, 1996 ; McGuire et Caldeira, 1993). Pour certains auteurs, même si les décisions des tribunaux sont toujours individuelles, elles dessinent de 
véritables politiques judiciaires qui ne sont jamais réductibles à des processus et mécanismes strictement juridiques (Canon et Johnson, 1998). L’implémentation des décisions peut s'avérer difficile, chaotique, incomplète, notamment parce que les acteurs judiciaires sont particulièrement démunis pour s’assurer de la mise en œuvre des décisions qu’ils prennent (Rosenberg, 1991 ; Canon et Johnson, 1998). Mais, en tout état de cause, ils interviennent de fait dans la définition du contenu même des politiques publiques et dans leur mise en œuvre concrète, notamment en établissant ce que l'administration peut faire et ne pas faire. Ce pouvoir du judicaire (en l’occurrence des tribunaux fédéraux américains) sur la conception et la mise en œuvre de politiques publiques et sur les administrations concernées a été particulièrement démontré dans le domaine de l’environnement. Ainsi dans une étude, fournissant par ailleurs une très abondante bibliographie sur ce thème, est soulignée l’influence des décisions des tribunaux fédéraux dans l'activité de l'agence de protection de l'environnement américaine dont les administrateurs sont susceptibles de devenir les exécutants... jusqu’à être dépossédés de leur pouvoir. L’orientation des politiques est définie par des tribunaux agissant en continu et n’hésitant pas, par exemple, à affaiblir le pouvoir des experts scientifiques au profit des services juridiques de l'agence de protection de l'environnement, ce qui témoigne d'une implication conjointe d'un judiciaire fortement incitatif et d'administrations dans la conception et la mise en œuvre des politiques publiques (O’Leary, 1989 ; voir également : Vig et Kraft, 1997).

Si cette influence croissante du judiciaire sur les politiques publiques est souvent démontrée à partir du cas américain, elle est également avancée pour d'autres pays. C’est ce qu’illustre l'exemple de la Colombie, particulièrement significatif d’un phénomène qui s’observerait plus largement dans l'ensemble de l'Amérique latine (Sieder, Schjolden, et Angell, 2005). C'est ainsi qu'en Colombie, à partir de 1998, la protection des droits sociaux par voie judiciaire a été sensiblement accrue, par exemple en matière de droit à la santé contre les organismes de 
sécurité sociale. De même la Cour Constitutionnelle a exercé une influence croissante en matière de politique économique, notamment en annulant des lois qui étendaient la taxe sur la valeur ajoutée à des produits de première nécessité, en ordonnant l'indexation partielle des salaires des fonctionnaires, en étendant le bénéfice de certains éléments de la pension de retraite à des groupes de population qui en étaient exclus, en interdisant la suppression d'avantages pour les retraités au motif que cette suppression remettait en cause des droits acquis des travailleurs (Uprimny Yepes, 2007).

Si l'on admet ce qui serait ainsi une plus grande implication des tribunaux dans la définition et la mise en œuvre des politiques publiques, celle-ci ne serait pas simplement quantitative (au sens où les tribunaux interviendraient plus massivement et plus souvent) mais aussi qualitative, au sens où l'action judiciaire prendrait une orientation nouvelle. Cette action judiciaire aurait quitté les rivages de l'adjudication, du jugement individuel et serait désormais susceptible de produire des résultats socialement désirables (Horowitz, 1977 ; Feeley et Rubin, 1998). En fait, la question importante qui mérite d'être posée ici est celle de savoir si cette présence de plus en plus affirmée du judiciaire dans le domaine des politiques publiques n’est pas liée à une véritable transformation du régime de la légalité, du statut du droit luimême. Pour Martin Shapiro, les pays de droit civil n’échapperaient pas à une relativisation de l'influence des codes pour laisser de plus en plus de place à un travail d'interprétation de telle sorte qu'il serait possible de dire que « loin de jouer avec un ensemble complet de normes juridiques préexistantes, les pays de droit civil, comme les pays de common law, dépendent de leurs juridictions pour légiférer par l'intermédiaire du travail de jugement » (Shapiro et Sweet Stone, 2002, p. 155). Le sens du droit et de la loi serait ainsi reconfiguré : la loi ne serait plus tant une référence immuable, elle acquérrait un caractère instrumental, orienté vers des objectifs de politique publique. La régulation juridique devrait alors incorporer, outre la règle 
de droit, la flexibilité, faite de négociations, de constructions de compromis, sans être forcément assujettie à des règles fixées au préalable (Feeley et Rubin, 1998, p.23).

De ce point de vue, les techniques du 'judicial policy making' deviendraient de plus en plus comparables à celles de toute autre agence gouvernementale. Une telle évolution de l'activité judiciaire pose évidemment la question de sa légitimité - puisque celle-ci reposait jusque-là sur la fidélité aux textes et sur la séparation des pouvoirs - mais plus encore celle de sa désirabilité, de la capacité et des conditions dans lesquelles les tribunaux peuvent et doivent assumer les sollicitations qui leurs sont adressées. La question est donc bien d'établir les « capacities and incapacities of courts as social policymakers » (Horowitz, 1977, p. 67).

$<$ IT2> 3.2- Les tribunaux comme acteurs faibles des politiques publiques

Tout un courant d'analyses critiques (Rosenberg, 1991), des débats et controverses (Canon et Johnson, 1999) autour de l'impact réel des décisions de justice témoignent en effet, à l’inverse des analyses précédentes, du fait que l’influence du rôle des juridictions dans le policymaking est loin d'être complètement démontrée. Au point que les juridictions américaines, en particulier la Cour Suprême, sont présentées par certains auteurs comme des «mouches de papier » (fly-paper courts) qui donnent l'illusion du changement alors même qu'elles sont structurellement peu capables de faire changer les choses, sauf dans des configurations très particulières qui, dans tous les cas, ne dépendent pas d'elles (Rosenberg, 1991). Une des manifestations de ce pouvoir limité est la difficulté pour la Cour Suprême de peser sur l’interprétation et la mise en œuvre de ses décisions par les juridictions inférieures à l’opposé donc d'un modèle pyramidal de judicial decision making (Reid, 1988), ceci d'autant que les modes de diffusion de l'innovation des tribunaux s’inscrivent dans des processus complexes, non linéaires ou forcément verticaux, où sont susceptibles d’interagir différents acteurs autres que les juges (Glick, 1992). Cette relativisation du pouvoir de la Cour Suprême tient certainement aussi aux conditions dans lesquelles elle produit ses décisions. Celles-ci 
apparaissent obéir à deux logiques : une logique juridique (culture du précédent, influence de la doctrine et de la jurisprudence...) et une logique extra-juridique (système de valeurs et positions idéologiques des juges, contexte social et politique...). La première de ces logiques survalorise la dimension libérale de la décision, la seconde (mise particulièrement en valeur par le courant du «judicial behavorialism », Gillman, 2001 ; Spaeth et Segal, 2000) insiste sur sa dimension conservatrice (George et Epstein, 1992).

De façon plus générale, outre le fait qu’ils ne sont pas maîtres d'une mise sur agenda de leurs interventions, qu’ils sont éventuellement dépendants des stratégies des forces partisanes (Gillman, 2002), les tribunaux seraient désarmés. Ils disposeraient de moyens limités et d’une faible capacité à faire exécuter leurs décisions, à s’assurer de leur mise en œuvre effective. La lenteur des processus de mise en œuvre contribuerait également à affaiblir l'impact de l'action des tribunaux. Par ailleurs, les autres branches du pouvoir pourraient elles-mêmes saboter les réformes initiées ou voulues par les tribunaux (Rosenberg, 1991).

Paradoxalement, les sollicitations croissantes dont la justice fait l'objet dans le domaine des politiques publiques contribueraient à dévoiler un peu plus le caractère traditionnel, archaïque, inadapté des méthodes de travail des tribunaux. Dans les années 1970, Donald Horowitz se penchait déjà sur l'étude des processus d'adjudication et sur leur adéquation aux nouveaux besoins des politiques publiques. Selon cet auteur, le problème concernait à la fois celui des données importantes pour la prise de décision, et la nécessité de concevoir des solutions flexibles, d'anticiper et de contrôler les effets des décisions, et ce, pour parvenir à ce que les tribunaux améliorent leur capacité de policymaking. Mais les difficultés des tribunaux en la matière, découlant de la nature même du travail d'adjudication, sont aussi à mettre en relation avec la capacité de policymaking et les performances des autres instances de gouvernement, ces dernières restant également problématiques (Horowitz, 1977). En fait, la question ici posée ne serait pas tant celle de la légitimité des tribunaux, du caractère démocratique ou non 
de leur action et du rôle qu'ils jouent. Elle serait surtout celle de la capacité et des conditions dans lesquelles les tribunaux peuvent et doivent assumer leur rôle dans la mesure où ils sont sollicités dans des sphères de plus en plus larges de l'action publique. Cela suppose, suivant cette perspective critique, que les tribunaux cessent de s'en tenir à une mobilisation sur le jugement, à une simple attente de soumission à leurs injonctions et qu'ils se préoccupent d'assurer un contrôle en continu de leur action, qu'ils soient d'abord préoccupés des fins plutôt que des moyens. Une contradiction apparaît alors entre le large spectre des décisions et de leur impact d’une part et les dispositifs réduits dans le cadre duquel ces décisions sont rendues d'autre part ; entre l'approche du tribunal soucieux de rendre une décision en termes de droits des parties et l'ampleur des innovations que le jugement est susceptible de provoquer au-delà de la seule affaire en cause. De façon significative, le processus judiciaire porte peu de considération au poids des options alternatives et au calcul des coûts ce qui explique qu’in fine, les décisions omettent généralement toute référence explicite aux coûts. Certes un retour éventuel au tribunal d'une affaire qu'il a traitée auparavant permet à celui-ci de prendre connaissance des conséquences éventuelles de ses décisions. Mais cette « limited policy review » ne saurait correspondre à une véritable supervision en continu telle qu'une agence administrative est en mesure de l'assurer. Bien entendu, les juges ne sont pas recrutés pour leur intérêt ou leur aptitude managériaux et ils ont le plus souvent peu de goût pour les procédures administratives. Il reste que les tribunaux ne devraient pas être dans l'incapacité de réajuster leurs décisions antérieures à la lumière des impacts que ces dernières ont éventuellement produits.

Un autre problème auquel les tribunaux auraient à faire face de plus en plus - au-delà de l'exercice de leur fonction traditionnelle, à savoir dire le droit à partir de cas concrets - serait d'évaluer le caractère plus ou moins représentatif des affaires qu'ils ont à traiter. Tirant la leçon de quatre cas longuement examinés, Donald Horowitz se préoccupe des façons dont les 
tribunaux pourraient prendre plus systématiquement en compte les faits sociaux dans une démarche où il ne voit pas de différence entre l'enquête juridique et ce qu'il considère comme une enquête de sciences sociales. Enfin, il resterait pour Donald Horowitz la question du suivi des décisions judiciaires. Il considère que si les tribunaux sont bien équipés pour appréhender clairement le passé et le présent, ils le sont nettement plus mal pour maîtriser le futur. En un mot, les tribunaux jouant un rôle de plus en plus important dans l'action publique manquent de ressources, s’appuient sur des dispositifs étroits et temporaires, pour pouvoir prétendre gérer des problèmes complexes et de grande ampleur. Finalement, pour Donald Horowitz, la spécificité du processus judiciaire est de mobiliser des ressources sociales à partir de recours individuels, alors que les tribunaux devraient pouvoir travailler avec des catégories générales qui transcendent les cas individuels. C’est là que réside pour l'essentiel l'incapacité des tribunaux à assumer un véritable travail de gouvernement (Horowitz, 1977).

Un certains nombre d'auteurs s'appuient sur ces constats critiques que l'ouvrage précurseur de Donald Horowitz établit pour, non pas nier le rôle du judiciaire dans le domaine des politiques publiques, mais pour le renforcer. Ainsi, de façon significative pour des tenants de cette position comme Malcolm Feeley et Edward Rubin, il doit être désormais attendu du gouvernement qu'il joue un rôle positif dans la réalisation du progrès social et la gestion de l'économie, ce qui suppose un régime de régulation non plus basé sur l’idée d'un «bon » gouvernement (avec un mécanisme maîtrisé d’équilibre entre des forces opposées) mais sur les efforts coordonnés d'une autorité centrale qui combinerait production des règles, activité de jugement (adjudication) et suivi des mises en œuvre de politiques (implementation) (Feeley et Rubin, 1998). Cela suppose toutefois pour ces auteurs que les tribunaux rompent avec la tradition qui veut qu'ils s'en tiennent à une mobilisation sur le jugement, à une simple attente d'une soumission à leurs injonctions sans se préoccuper d'assurer un contrôle en continu de leur action (Feeley et Rubin, 1998). Pour eux, un Judicial Policy Making doit 
s'inscrire dans la conception moderne de l'Etat qui exige une active policy making judiciary et une conception du droit plus « active ». La réflexion de ces auteurs s’appuie sur une étude des décisions de la justice fédérale américaine de 1965 à la fin des années 1990. Parler de policy making à propos de la justice, c'est considérer que l'activité judiciaire ne se limite pas au jugement, à l'adjudication, mais qu'elle est susceptible de produire des résultats qui sont socialement désirables. Mais le cœur de la démonstration est ici que, pour ces auteurs, la croissance d'un Etat bureaucratique exige la multiplication d'instances de régulation, d'agences indépendantes, ce qui n’a plus rien à voir avec la structure en trois pouvoirs de Montesquieu (Feeley et Rubin, 1998). Une telle évolution nécessite aussi que soit consacrée une nouvelle conception du droit et de la référence aux règles juridiques dans l'activité judiciaire. On doit désormais admettre le caractère instrumental, policy-oriented de la règle de droit comme conception dominante. C’est une conception managériale de l'exercice du gouvernement qui s’impose, dans laquelle la fonction judiciaire occupe un rôle, c'est-à-dire une conception globale, unifiée de l'exercice du pouvoir public, laquelle rend caduc le modèle du XVIIIe siècle de la primauté du contrôle du pouvoir par la séparation des pouvoirs. Ce déclin du modèle traditionnel de la séparation des pouvoirs doit s'effectuer au profit d'une conception de l'exercice du pouvoir efficace et cohérent. Pour ces auteurs, il faut reconnaître la pertinence de nouvelles doctrines juridiques et abandonner l'idée que la règle de droit implique la fidélité à tous les principes légaux préexistants (Feeley et Rubin, 1998). L’Etat de droit demeure un principe important et valide mais c’est un principe qui a été transformé par la réalité administrative et la théorie sociale moderne: d'une sollicitation de règles préétablies, il s’est transformé en une inscription des règles dans l'action publique. L’étude du judicial policy making devient alors l'étude d'un nouvel ordre légal et d'un nouveau mode de gouvernement dont il participe : «The incrementalism of judicial policy making means that it must incorporate both bodies of law at the same time that it produces change " (Feeley et 
Rubin, 1998, p. 356). Les juges doivent devenir des policy-makers parce que le policy making est devenu le principal mode caractérisant l'action gouvernementale (Feeley et Rubin, 1998). Ainsi le judicial policy making serait révélateur de l'avènement d’une conceptualisation nouvelle à la fois du droit et de l'action gouvernementale (Feeley et Rubin, 1998).

Ce qui caractérise en grande partie cette littérature apparaît ici clairement: sa double inscription dans le registre de l'affirmation que des changements ont lieu et dans celui de l'évaluation de la nature plus ou moins désirable de ces changements. Les auteurs se font les avocats d'une judiciarisation qui serait synonyme d'une transformation du statut du droit et de son rapport à l’action politique.

La forme engagée et prescriptive de ces analyses n’est pas mieux démontrée a contrario que par la recherche rigoureuse effectuée par Gerald Rosenberg sur des décisions judiciaires dont il reconstitue minutieusement le processus en confrontant les données empiriques disponibles aux arguments avancés par les tenants d'une « conception dynamique » du rôle de la justice dans le changement social. Il conclut que le rôle des tribunaux dans le changement social est au bout du compte limité et que bien des réformes interviennent indépendamment et en dehors du système judiciaire, la seule influence qui peut alors être accordée aux tribunaux se limite aux cas où ils inscrivent leur action dans un cadre où interviennent d'autres facteurs, d'autres forces qui concourent à un changement dont la justice ne prend alors qu'une petite part (Rosenberg, 1991). Dans la même veine, un inventaire méthodique et fondé empiriquement du rôle de la Cour Suprême américaine et des juridictions fédérales dans le domaine des droits de l'enfant, particulièrement ceux issus des classes pauvres ou des minorités, aboutit au constat que ce rôle, à l'instar de celui de l'Etat fédéral, reste particulièrement limité (Mezey, 1996). Au-delà d'un certain volontarisme affiché, l'impact des tribunaux sur ce secteur de politiques publiques resterait faible. 


\section{$<\mathrm{IT}>4$ - Les mises en œuvre de la légalité comme enjeux pour la démocratie}

L’exemple du rôle de la justice dans le domaine des politiques publiques suffit à révéler que les enjeux évoqués ne se situent pas seulement au niveau de ce que seraient les recompositions du travail gouvernemental et le déplacement progressif du rôle de la justice. Les questions qui sont posées concernent bien la nature même du politique et de ses métamorphoses, celle de la remise en cause de l'équilibre traditionnel des pouvoirs législatif, exécutif et judiciaire, celle des formes de réalisation de la démocratie. C’est certainement l'ampleur de ces enjeux qui explique le caractère très contrasté des approches qui sont développées autour du phénomène de judiciarisation, ce que nous pourrions mettre en scène sous la forme de l'opposition entre une vision enchantée et une vision critique de ce qui serait l'extension du rôle de la justice dans l'accomplissement du politique.

$<$ IT2> 4.1- La vision enchantée

Toute une partie de la littérature sur la judiciarisation est consacrée à souligner l'accroissement du rôle des Cours Suprêmes, plus largement celui des tribunaux. La judiciarisation serait un facteur de progrès social, permettrait que soient plus et mieux défendus les droits de l'homme, les droits des plus faibles... Ce rôle de la justice dans l'égalisation des conditions des membres de la communauté politique est non seulement affirmé mais aussi célébré par cette littérature. C’est ainsi, par exemple, que l’institution de la Cour Européenne de Justice et de la Cour Européenne des Droits de l’Homme est supposée avoir contribué à la promotion des droits sociaux, prenant la forme de droits supranationaux, notamment pour les citoyens les plus vulnérables (Conant, 2006). De façon parfaitement illustrative, Charles Epp avance l'idée d'une «Rights Revolution» caractérisée par le renforcement des garanties constitutionnelles pour les droits individuels et l'indépendance 
judiciaire, le leadership assuré par des juges activistes (particulièrement ceux des Cours Suprêmes) utilisant leurs pouvoirs pour transformer la société et promouvoir un accroissement de la conscience des droits dans la culture des sociétés (Epp, 1998). Pour cet auteur, les EtatsUnis occupent une position de pointe dans cette «Rights Revolution ». Si auparavant et jusqu’à peu, le recours à la justice aux Etats-Unis était le fait des citoyens les plus puissants, la « Rights Revolution » aurait permis aux citoyens ordinaires d'accéder à ces bienfaits de la justice. La Rights Revolution se définit alors comme un processus de production et d'extension de nouveaux droits civils et de libertés qui comporte trois composantes : une attention de la justice aux nouveaux droits, un soutien de la justice aux nouveaux droits, et une politique de renforcement de ces nouveaux droits (Epp, 1998).

Pour étudier cette «Rights Revolution », Charles Epp entreprend une recherche comparative portant sur quatre pays : les Etats-Unis, l’Inde, la Grande Bretagne et le Canada, au cours de la période 1960-1990. Dans chacun des pays, deux domaines sont analysés : la défense des détenus et les droits de leurs défenseurs d'une part, les droits des femmes d'autre part. Le succès de la « Rights Revolution » aux Etats-Unis s’expliquerait pour Charles Epp par la mise en place d'une ambitieuse politique en faveur de la « litigation » et par l'accroissement d'une structure de soutien («support structure ») permettant la mobilisation juridique («legal mobilization »). Cette «structure de soutien» suppose selon C.Epp des organisations de défense des droits («rights advocacy organizations ») qui sont équipées juridiquement, un large éventail de sources de financement et des efforts au niveau fédéral pour encourager les coalitions de défense des droits («rights advocacy»). C’est grâce à cette structure de soutien particulièrement développée aux Etats-Unis, que la Cour Suprême a pu bâtir la révolution américaine des droits. A contrario, l’Inde n'est pas dans la même situation, même si la Cour Suprême de ce pays est, aux yeux de l'auteur, une des plus actives du monde. Cette situation s’expliquerait par la faiblesse de la structure de soutien nécessaire à la mobilisation juridique. 
La situation de la Grande Bretagne, quant à elle, est plus complexe car si, au départ, rien ne permet d'escompter une «Rights Revolution », le développement d'une support structure permet de comprendre le fait que l'«Appellate Committee» de la Chambre des Lords britannique soit passé du traitement de conflits principalement d'ordre économique à un contentieux relevant du droit public et notamment des revendications de droits («rights claims »). Il reste toutefois la relative faiblesse des groupes de défense des droits et des sources de financement. En ce qui concerne le Canada, Charles Epp reconnaît l'influence de la Charte des droits et libertés de 1982 sur la Révolution des droits. Il estime toutefois que le facteur décisif n’est pas la Charte dont l’influence est de son point de vue surestimée mais là encore l'effet d'une structure de soutien permettant la mobilisation juridique, dont une des manifestations est une pression croissante venue de l'extérieur du judiciaire. En conclusion, Charles Epp souligne que les droits ne sont pas donnés mais gagnés grâce à des actions collectives suscitées par une société civile dynamique et l’octroi de fonds publics.

C’est dans le même esprit qu'un auteur indien souligne le rôle croissant de la Cour Suprême indienne dans la promotion des principes démocratiques, d’une conception plus substantive de l'égalité ainsi que du contrôle de la régularité des processus électoraux (Mehta, 2007). La Cour Suprême a notamment promu une innovation: le «Public Interest Litigation » permettant à tout citoyen de se pourvoir en justice dans la mesure où il aurait été victime « of an alleged evil » ou d'une injustice. Mais l'auteur reconnaît néanmoins que ce rôle de la Cour Suprême est altéré par de profondes ironies : l'accès à la justice ordinaire qui reste difficile, la contradiction entre l'établissement de la Cour Suprême comme un forum pour résoudre les problèmes de politique publique et le peu de clarté des principes structurant son action.

La vision positive du phénomène de la judiciarisation a sa crédibilité renforcée, non pas seulement parce qu'elle est nourrie par ce qui serait un changement des pratiques des juridictions, mais également par cette réalité qui s’affirme de nouveaux rapports des citoyens 
eux-mêmes avec la justice. Dans une logique de démocratisation et d'implication démocratique dans l'activité judiciaire, notamment au niveau européen (voir par exemple : Cichowski, 2004 ; 2006a ; 2006b), les mouvements sociaux sont perçus comme des acteurs dynamiques de la judiciarisation. L'un des ouvrages marquants de cette orientation est celui de Michael McCann : Rights at Work. En effet, dans cet ouvrage, l'auteur s’intéresse à l'utilité de la loi dans le renforcement du pouvoir des citoyens marginalisés ou même des citoyens ordinaires. Face à une représentation de la loi qui suggère un ordre hiérarchique, Michael McCann entend démontrer que, dans les mains de citoyens en rupture, la loi peut être une source de désordre et d’égalitarisation des conditions. Cette conception s’inscrit dans une approche et une définition de l'action collective qui met l'accent sur les luttes contre les hiérarchies et les injustices. Dans cet esprit, la mobilisation du droit participe directement d'un processus politique de contestation d'un ordre établi. Comme le souligne cet auteur dans un article plus récent, le droit fournit «à la fois des principes normatifs et des ressources stratégiques pour la conduite des luttes sociales » (McCann, 2004, p. 508). Il est intéressant de noter qu'il ne s’agit pas forcément ici de gagner un procès mais de faire avancer une cause «dans l'ombre de normes juridiques favorables et de la menace d'une intervention judiciaire » (McCann, 2004, p. 515). L’objet support de la démonstration de Michael McCann, ce sont les actions collectives menées par les juristes des syndicats américains qui portent sur l'égalité de salaire («pay equity») concernant principalement les femmes mais aussi les individus souspayés ou sous-valorisés au motif de leur appartenance à des minorités sexuelle ou raciales, ces actions collectives ayant abouti à une décision favorable de la Cour Suprême. Dans cette perspective, la loi est conçue comme une pratique sociale et participe d'un ordre légal pluraliste avec une multitude de traditions juridiques « indigènes » et relativement autonomes, issues de nombreuses sous-cultures et instances au sein de la société. La loi est donc ici susceptible de structurer des stratégies potentielles de résistance et de prendre des formes 
multiples suivant les différents terrains de luttes sociales (McCann, 1994). L’objectif de recherche s'inscrit dans un engagement de longue date pour analyser les promesses et les limites des actions légales menées dans le cadre de combats assumés par des mouvements sociaux progressistes, plus généralement, les luttes contre la hiérarchie et la domination. Michael McCann souligne combien ses analyses et son cadre de recherche s'inspirent et empruntent aux travaux féministes. Il considère que son projet est en rupture avec les analyses classiques sur le droit, ce qui le conduit à qualifier les activités juridiques auxquelles il s’intéresse de « bottom up jurisprudence » (McCann, 1994, p. 22).

Selon lui, une telle orientation est à l'opposé de celles de chercheurs qui ont accordé peu d'importance à l'usage d'instruments juridiques dans les luttes pour le «pay equity» alors que les luttes des femmes travailleuses ont montré l'importance que pouvait avoir l'outil juridique dans ces luttes (McCann, 1994). C’est ainsi que, toujours selon Mc Cann, des décisions de justice ont changé les rapports de force entre des travailleuses et leurs employeurs, faisant ainsi croître les attentes et les espoirs envers de possibles réformes (McCann, 1994). De telle sorte que, selon cet auteur, on peut dire que les décisions judiciaires et les normes juridiques ne sont pas auto-générées mais qu'elles constituent des ressources potentielles qui peuvent être ou non mobilisées dans des actions pratiques (McCann, 1994). Les actions pour le «pay equity» ne découlent pas des constructions logiques de juristes ou de juges. Elles résultent de normes et de pratiques qui organisent la vie de travail d'un grand nombre de femmes (McCann, 1994).

Ce que souligne cet auteur, c’est le fait que les bénéfices d'actions qui utilisent le droit et la justice comme ressources vont au-delà des effets concrets appréhendés sous la forme de droits acquis. Ils offrent des ressources plus larges aux mouvements sociaux qui les ont mis en oeuvre. De ce point de vue, même des réformes limitées peuvent favoriser de fortes avancées qui ne se limitent pas à des cas individuels (McCann, 1994). En effet, c'est un pouvoir 
collectif qui se constitue à travers l'intensification des liens parmi les militants et les groupes politiques (McCann, 1994), ce que les militants désignent eux-mêmes comme le renforcement du collectif par l'intensification d'une solidarité (McCann, 1994). Ainsi que le montrent les propos tenus par les femmes interviewées par Michael McCann, la mobilisation a pour effet d'accroître la conscience des droits chez les femmes actives aux Etats-Unis et de renforcer leur sentiment de solidarité (McCann, 1994). Un mouvement qui avait ainsi un objectif limité au départ ouvre des perspectives pour une expansion des droits des femmes, au-delà de la question posée initialement (McCann, 1994), et pour un développement d'une conscience de leurs droits («rights consciousness ») (McCann, 2004, p. 518).

Une littérature abondante s’inscrit dans le droit fil de ces analyses de Michael McCann et confirme l’importance qui est accordée à ces mobilisations collectives dans les explications du phénomène de judiciarisation. Nous pouvons ainsi citer cette étude canadienne portant sur les mouvements gays et lesbiens qui insiste sur le rôle des tribunaux dans la reconnaissance des droits de ces populations (Smith, 2005 ; voir également pour les Etats-Unis, sur le même thème : Andersen, 2005). Pour Miriam Smith, l'accroissement du pouvoir judiciaire en la matière a certes été favorisé par la Charte canadienne des droits et libertés de 1982 (dans le sens de cette même vision positive de la Charte, voir également : Morton, 2002). C’est dans ce contexte que des leaders activistes ont fait le choix du recours aux tribunaux comme stratégie politique, en considérant que la référence aux droits civils pouvait être un moyen privilégié pour consacrer l’idéologie de la «gay liberation». Les observations les plus centrales de cette démonstration concernent :

-l'avènement, à la suite de cette mobilisation juridique et judiciaire, d'une nouvelle conscience des droits ;

- l’influence décisive dans le processus des professionnels du droit qui assurent un relais : « l’organisation du mouvement gay et lesbien est passée de groupes locaux et urbains 
à un réseau de défense des droits pan canadien dominé par des professionnels du droit et des organisations de défense juridique» (Smith, 2005, p. 346). Ce phénomène est une des expressions du «cause lawyering » (Sarat et Scheingold, 2006). Il renvoie également à ces processus historiques déjà observés de réinscription des professionnels du droit dans des mouvements sociaux dont des représentants de la société civile avaient eu l'initiative et, pour un temps, la maîtrise (voir par exemple les tribunaux de famille sous la Révolution française : Commaille, 1989) ;

-le déplacement de l’espace politique vers l'espace juridico-judiciaire pour traiter d’un problème qu'il revient logiquement au politique de traiter (Smith, 2005). Ainsi, selon Miriam Smith, l'ouverture politique fournie par la Charte a fortement incité le mouvement gay et lesbien à s’organiser dans le cadre de réseaux juridiques structurés par les professionnels du droit (Smith, 2005).

Suivant la même logique mais dans une optique un peu différente, de nombreux auteurs soulignent le fait que certains groupes d’intérêts ont développé de nouvelles stratégies politiques, qui passent précisément par des recours en justice. Barry Holmström donne ainsi l'exemple des différentes requêtes présentées devant la Cour de Strasbourg contre la Suède. Les cas de condamnation de la Suède ont été portés directement ou plus discrètement par des groupes d’intérêt comme l’Association suédoise de protection des intérêts du logement et de la construction ; la Fédération des fermiers suédois ou des intérêts liés au secteur des affaires. Ces groupes de pression nationaux utilisent ainsi la scène judiciaire internationale et le recours à la ressource juridique pour tenter de promouvoir et de faire triompher leur cause (Holmström, 1994).

\section{$<$ IT2> 4.2- La vision critique}

Michael McCann a largement souligné le caractère ambigu et contradictoire du droit dans les sociétés libérales, ce qui nous semble particulièrement emblématique de la difficulté à donner 
un sens au phénomène de judiciarisation, tantôt danger, tantôt panacée dans l'accomplissement et la mise en œuvre du politique. En effet, pour McCann, les normes juridiques et les institutions ne garantissent pas la justice non plus qu'ils ne sont un simple obstacle dans la recherche d’une société plus juste. Ceux qui ne reconnaissent que l’une ou l'autre option sombrent dans la facilité (McCann, 1994). En fait, selon cet auteur, on peut considérer que la domination est bien davantage susceptible d'être remise en cause dans les sociétés libérales plus que dans bien d'autres régimes. La ressource juridique est de ce point de vue cruciale. Et les radicaux qui relativisent ces possibilités de changement ne rendraient pas service aux possibilités de changement social affirme McCann, visant notamment un auteur comme Rosenberg (McCann, 1994). Mais pour trancher en la matière, Michael McCann considère qu’il ne suffit pas de débattre : il faut entreprendre des recherches où la théorie est fondée sur un travail empirique pour saisir le poids des usages des ressources juridiques dans les luttes sociales (McCann, 1994).

Plusieurs auteurs développent une perspective critique d'une autre nature. Ils soulignent la difficulté de concilier « l’activisme judiciaire » avec la démocratie et le danger que représente le transfert vers le judiciaire de questions qui sont traditionnellement du ressort de politiques élus et d'institutions fonctionnant suivant le principe de la majorité. Charles Epp, par exemple, évoque dans son ouvrage les débats politiques que suscite l'accroissement du pouvoir judiciaire. Pour lui, la « Rights Revolution » est au coeur de la relation entre les droits et la démocratie mais il rappelle que pour les auteurs qui ont adopté un positionnement critique à l'égard de ces nouveaux droits considèrent que le pouvoir judiciaire s’est saisi de ce mouvement pour accroître son propre pouvoir en dehors de tout contrôle et a de ce fait affaibli le processus démocratique, fondé sur les principes électif et majoritaire. Charles Epp cite ainsi Robert Bork pour qui «la «Rights Revolution » est «le transfert dans la Constitution des principes de la culture libérale qui ne peuvent être mis en œuvre démocratiquement » (Epp, 
1998, p. 4). Ainsi la Constitution aurait subi une profonde transformation, passant d'un mécanisme de limitation du caractère arbitraire du pouvoir gouvernemental à une source d'exercice de l'arbitraire du pouvoir judiciaire. Mais il rapporte aussi les réflexions de défenseurs de ces nouveaux droits qui avancent, après avoir été méfiants, que l'extension d'une grande partie de ces droits va dans le sens d'un renforcement de la démocratie. Charles Epp estime quant à lui que cette crainte exprimée à l'égard des juges et de la justice est exagérée et que le souci formulé par rapport à la démocratie est mal fondé. Il avance comme argument principal le fait que la «Rights Revolution » dépende d’un large soutien permis par une démocratisation de l’accès à la justice.

Les commentaires de Miriam Smith sur les effets de la Charte canadienne des droits et libertés de 1982, dans le cadre de son analyse sur le mouvement gay et lesbien, représentent une des nombreuses réflexions portant sur les avantages tirés par les mouvements sociaux d'un recours à la justice (voir également les mobilisations des mouvements féministes en la matière, par exemple : Morton et Allen, 2001 ; Manfredi, 2004 ; pour l'influence d'un « constitutionnalisme supranational » européen favorisant le recours à la justice des mouvements féministes, voir : Cichowski, 2004). Mais surtout les analyses de Miriam Smith s’inscrivent dans la controverse de grande ampleur suscitée par cette Charte. Pour elle, le caractère partisan des réflexions qui lui sont consacrées est patent. La gauche comme la droite insistent sur les aspects négatifs de cet accroissement du pouvoir judiciaire. La gauche déplore que l'engagement dans le droit présente un risque de dépolitisation du mouvement social. Quant à la droite, elle regrette que la Charte, en renforçant le pouvoir du droit et de la justice, permette à ceux qui en sont des professionnels d'entrer sur le terrain de la politique et de la production de programmes publics, excédant ainsi ce qu'elle considère comme étant un juste exercice de leur fonction juridique. 
Mais ce sont les propos d'un autre auteur qui témoignent le plus du caractère passionnel pris par ce débat sur la Charte des droits et plus largement sur le phénomène de judiciarisation cette réaction étant particulièrement exemplaire pour notre propre analyse. Pour Rainer Knopff (Knopff, 1998), « la démocratie représentative est menacée par les forces constituées à la fois par le populisme et la politique judiciarisée des droits » (Knopff, 1998, p. 683), ceci dans la mesure où, précisément, la politique des droits déplace le pouvoir de la représentation élue vers des juges appointés » (Knopff, 1998). « Le danger du populisme et de la politique judiciarisée des droits repose sur un appel mystique au-delà de la politique ordinaire » (Knopff, 1998, p. 684) que l'auteur désignera dans la suite de son article par l'expression de « tentation théocratique ». "Dans une démocratie libérale, [celle-ci] cache ses ambitions derrière les bannières à la fois des droits et du peuple » (Knopff, 1998, p. 704), ce qui représente une menace certaine pour la démocratie. Un autre auteur n’est pas loin de tenir la même position quand il souligne combien la promotion de la Cour Suprême canadienne au nom de la défense des intérêts de groupes sociaux «désavantagés » dissimule en fait les stratégies de renforcement de leur pouvoir des leaders de groupes politiques parfaitement implantés dans l’Etat (Brodie, 2002).

La question posée bascule dès lors vers celle du déséquilibre des pouvoirs que provoquerait le phénomène de judiciarisation. C’est bien en ces termes que Pratap Bhanu Mehta pose le problème. Pour cet auteur, l'accroissement du pouvoir de la Cour Suprême en Inde s'est fait en usurpant des fonctions qui reviennent à l’Exécutif en marginalisant la représentation politique sans justifier clairement ce qui légitime l’autorité judiciaire. La légitimité de ce pouvoir judiciaire est ainsi interrogée : « qu’est-ce qui légitime l’activisme judiciaire et n’en fait pas la manifestation d'un excès de pouvoir mais l'expression d'une juste autorité ?» (Mehta, 2007, p. 80) car il n'y a aucune raison de penser que les juges, plus que les politiciens, protégeront toujours les libertés. 
De ce point de vue, les analyses de Ran Hirschl vont encore plus loin puisqu'elles laissent supposer de véritables stratégies des élites et une connivence entre élites politiques, économiques et judiciaires pour favoriser le mouvement de judiciarisation. Dans son ouvrage Towards Juristocracy, cet auteur rejoint le constat si souvent avancé selon lequel on observerait à travers le monde un mouvement de transfert d’une ampleur sans précédent des institutions représentatives vers le judiciaire, national et supranational. C’est ce phénomène que Ran Hirschl qualifie de juristocracy. Comme souligné plus haut, pour certains analystes, ce mouvement va dans le sens d’un progrès social, politique, ou témoigne d'une évolution vers une démocratie plus assurée et une célébration sans réserve des droits de l’homme. Mais, à partir d’une recherche comparée sur plusieurs pays (Canada, Nouvelle-Zélande, Israël, Afrique du Sud), Ran Hirschl avance une thèse qui contredit fortement ces interprétations. Pour lui, le renforcement du pouvoir de la justice via un mouvement de constitutionnalisation témoigne d’une volonté de préservation d'intérêts hégémoniques. Il s’inscrit dans un contexte de luttes sociales, politiques et économiques qui structurent le système politique et ne peut par conséquent être compris isolément. Ainsi le déplacement de l’autorité politique des arènes de prise de décision fondées sur le principe de la majorité vers les arènes judiciaires doit être attribué à des élites politiques et économiques qui considèrent que leurs intérêts sont ainsi mieux préservés ${ }^{5}$. Pour Ran Hirschl, ceux qui sont prêts à payer le prix du renforcement du pouvoir judiciaire sont ceux qui considèrent que leur position relative ou absolue pourrait être améliorée grâce à la «juristocracy» (Hirschl, 2004). Cette stratégie se développe particulièrement avec trois groupes clefs : les élites politiques qui voient leur hégémonie et leurs intérêts menacés par de nouveaux groupes sociaux et prétendent revendiquer un surcroît

\footnotetext{
${ }^{5}$ La question de la judiciarisation renouvelle ainsi le débat classique sur le fait que ce seraient les élites qui tireraient le mieux parti du recours à la justice, ce qui disqualifierait l'idée suivant laquelle la justice pourrait contribuer au changement social : voir la célèbre analyse de Marc Galanter (Galanter, 1974) ; voir également l'ouvrage consacré postérieurement à un bilan critique de cette analyse (Kritzer et Silbey, 2003) ; voir enfin une analyse portant sur une juridiction fédérale et qui conclut que les juridictions peuvent être plus réceptives que d'autres arènes politiques à des revendications émanant de groupes défavorisés mais qu'elles sont aussi utilisées par des groupes sociaux puissants pour renforcer leurs gains acquis politiquement (Olson, 1990).
} 
de démocratie tout en en contournant les procédures habituelles ; les élites économiques qui perçoivent la constitutionnalisation des droits, notamment ceux concernant la propriété, la mobilité et les droits de l'emploi comme un moyen de peser sur l'action du gouvernement et de promouvoir un marché sans contrainte (a free market) et un agenda favorable aux affaires ; les élites judiciaires et les tribunaux supérieurs au niveau national enfin, lesquels cherchent à renforcer leur influence politique et leur réputation internationale. En un mot, selon Ran Hirsch, ce sont «des innovateurs stratégiques dans le domaine juridique (strategic legal innovators) - [c'est-à-dire] les élites politiques en association avec les élites économiques et judiciaires qui ont des intérêts compatibles - [qui] déterminent le timing, l'ampleur et le contenu des réformes constitutionnelles » (Hirschl, 2004, p. 12).

Pour Ran Hirschl, le cas israëlien fournit un excellent exemple de montée en puissance de la Cour suprême. Martin Edelman avait lui-même souligné une augmentation quantitative très sensible des recours auprès de cette juridiction. Cet auteur estimait ainsi que, de 1956 à 1987, le nombre de cas soumis avait augmenté de 632 \% alors que, dans le même temps, la population s’était accrue de 230 \% (Edelman, 1994). En 1987, la Cour avait reçu 1466 pétitions, c’est-à-dire sept fois plus que trente-deux ans plus tôt (Edelman, 1994). Cet auteur observait en outre que la Cour Suprême israélienne se livrait de plus en plus à une interprétation libre de la Constitution (même s’il n’y a pas de Constitution écrite), dans l'invocation large de l'Etat de droit et de principes du droit naturel. Pour Martin Edelman, le système politique israelien aurait ainsi changé :cette juridiction serait aujourd'hui devenue un acteur politique majeur, alors qu'à la fondation d'Israël elle ne jouait qu'un rôle marginal. Elle serait en fait passée d'un rôle proche de celui de la Chambre des Lords dans le système britannique à un rôle davantage comparable à celui de la Cour Suprême américaine (Edelman, 1994). Mais Ran Hirschl, conformément à la position critique qu'il tient sur cette expression exemplaire d'une judiciarisation, avance une explication en trois points pour analyser 
l'accroissement voulu du pouvoir judiciaire israëlien : l’hégémonie des élites est menacée par des groupes périphériques et leurs orientations politiques; le pouvoir judiciaire bénéficie d’une réputation élevée de droiture et d’impartialité ; les juridictions sont enclines à statuer en accord avec les conceptions idéologiques et culturelles dominantes. (Hirschl, 2004).

Cette démonstration est reprise dans un article où Ran Hirschl souligne que la constitutionnalisation des droits en Israël est le produit d'une alliance stratégique entre des élites dominantes, leurs représentants politiques et les professionnels du droit (Hirschl, 2001). Il montre en particulier que la Cour Suprême israëlienne partage les valeurs d'une élite urbaine appartenant à la bourgeoisie ashkenaze, cette proximité est accrue par le fait que cette dernière exerce de fait un contrôle sur les nominations (Hirschl, 2001). Cette influence du pouvoir judiciaire est d'autant plus susceptible de s'exercer qu'elle est renforcée par la croyance, répandue dans l’opinion publique, en l’impartialité de ce dernier (Hirschl, 2001). Hirschl note toutefois que les représentants des minorités périphériques tendent à s’opposer de plus en plus à ce pouvoir judiciaire. En occupant une place de plus en plus importante, ils essaient d'imposer le retour aux arènes de prises de décisions politiques, en conformité avec le principe de majorité.

Faisant le bilan de ses observations, Ran Hirschl souligne, dans son ouvrage, que, dans les quatre pays étudiés, l'accroissement du judiciaire est fondé sur une conception étroite des droits, une référence appuyée à la conception individualiste de Locke, et une conception antiétatique et dyadique des droits constitutionnels. Il s’agit d’abord de protéger la sphère privée (humaine et économique) des pressions du collectif (souvent défini comme étant l'Etat et ses instances de régulation). Selon Ran Hirschl, l'Etat est perçu dans ces pays par les cours supérieures comme une menace pour la liberté humaine et l'égalité. Pour lui, le discours du droit sur les droits est imprégné de tous les principes du néolibéralisme dans ses dimensions sociales et économiques comme l’individualisme, la dérégulation, la « commodification » des 
services publics et la réduction des dépenses publiques, ceci inspiré par l'antiétatisme, la recherche d'une atomisation sociale, et la stricte protection de la sphère privée. Il s'agit dans cet esprit de préconiser une justice procédurale, moins d’intervention étatique sur la sphère privée ainsi que de manifester de l'hostilité à l'égard de toute demande d'égalité fondamentale, de régulations étatiques et de droits des travailleurs. (Hirschl, 2004). Ran Hirschl souligne ainsi une tendance globale, perceptible dans les quatre pays étudiés, à aller vers un néolibéralisme économique et social, avec une propension à l'affaiblissement d’une conception keynésienne du welfare state au profit de politiques orientées vers plus de marché et moins d’Etat (Hirschl, 2004). Bien entendu, pour cet auteur, ces évolutions ne vont pas dans le sens d’un renforcement d'une justice distributive ou de l'amélioration de la condition de groupes défavorisés (Hirschl, 2004).

Dans sa conclusion, Ran Hirschl suggère que ce processus de déplacement vers le judiciaire est susceptible de s’accentuer avec la création de juridictions supranationales (il prend l'exemple de la Cour européenne de justice) ou même d’instances semi autonomes comme les banques centrales nationales ou la banque européenne ou encore les traités de commerce transnationaux. Tout ceci témoignerait d'une tendance vers l'institution de procédures quasi judiciaires dans différents secteurs, ainsi que le transfert d'arènes fonctionnant sur le principe de la majorité politique vers des corps de producteurs professionnels de politiques semi autonomes (Hirschl, 2004).

La question générale qui est finalement posée est bien celle des rapports entre judiciarisation et démocratie. De façon exemplaire, dans la filiation intellectuelle de Ran Hirschl, Alexei Trochev développe une réflexion sur l'institution de cours constitutionnelles tout au long des années 1990 dans les régions appartenant à la Russie (Trochev, 2004). Pour lui, l’institution de ces cours participe d'une stratégie de politiciens visant à préserver leur pouvoir y compris pour échapper à « la tyrannie des vainqueurs d'élections » et à lutter contre les aspirations 
issues de majorités politiques. Le titre de l'article porte toute la signification de la thèse défendue : «Less Democracy, More Courts ».

Au terme de ce bilan - encore provisoire - de la littérature internationale sur le phénomène de judiciarisation, les incertitudes demeurent en ce qui concerne la réponse à la question : d'où parlent les auteurs ? Nous sommes effectivement dans un domaine où existe une particulière propension à tenir pour vrai ce qui est seulement souhaité. C’est sans doute ce qui explique que les observations apparaissent extraordinairement contrastées, notamment sur ce qui tient à la réalité du phénomène et ce qui tient aux représentations dont il est l’objet ; sur son ampleur et sur ce qui relève de singularités tenant à des traditions juridiques, à des configurations nationales spécifiques inscrites dans l’histoire, etc. ; sur les interprétations qu’il suscite et qui peuvent aller de la célébration à la dénonciation.

Comme nous l'avons vu, ce qui est souvent évoqué par les auteurs dans leurs analyses, c’est une judiciarisation qui impliquerait un déplacement du pouvoir du Législatif, de l’Exécutif vers le judiciaire ${ }^{6}$. Par exemple, pour Alec Stone Sweet, les tribunaux agissent de plus en plus comme des instances législatives et la distinction entre la production des lois par la voie parlementaire et par la voie judiciaire se dissout complètement, en particulier au niveau européen où « les législateurs européens gouvernent avec les juges » (Stone Sweet, 2000, p. 193). Prenant l'exemple du Conseil Constitutionnel en France, ce même auteur parle d'une « troisième Chambre du Parlement » (Stone, 1992). Même si ce constat est parfois nuancé ou discuté (voir, par exemple : Koopmans, 2003; Shapiro, 1994 ; Sunkin, 1994), ce déplacement

\footnotetext{
${ }^{6}$ A l'idée d'un déplacement, il convient d'ajouter celle d'une modification des relations entre ces instances si l'on se réfère notamment au fait que certaines analyses soulignent l'existence de stratégies de l'Exécutif ou du Législatif par rapport au judiciaire. Par exemple, la possibilité qu'une minorité politique saisisse une Cour constitutionnelle peut amener la majorité politique à anticiper et à construire préventivement des compromis (Vanberg, 1998).
} 
de pouvoir, observé, supposé, souhaité ou dénoncé, apparaît bien comme un enjeu majeur au sens où il situe la question de la judiciarisation au cœur du politique. Rien ne le montre mieux que le projet théorique d'Alec Stone Sweet, l'un des auteurs marquants de cette littérature. Son projet repose sur l’idée de l'avènement d'un nouveau régime de gouvernance. Pour cet auteur, la judiciarisation dans le traitement des conflits est le processus à travers lequel un TDR (triadic dispute resolution) apparaît, stabilise et développe une autorité, celle-ci détenue par une structure normative chargée de gérer les échanges dans une communauté donnée (Shapiro et Stone Sweet, 2002, Stone Sweet, 1999). Pour Alec Stone Sweet, cette triade constitue un mécanisme essentiel du changement politique. En fait, le passage de la dyade à la triade correspondrait à l'avènement d'une forme particulière de gouvernance. Il s'agit de réguler les comportements et de maintenir la cohésion sociale dans un contexte de changement. L'introduction d'un nouveau tiers, qui se manifeste par le phénomène de judiciarisation «structure progressivement les comportements stratégiques des acteurs politiques engagés dans des interactions les uns par rapport aux autres » (Stone Sweet, 1999, p. 71) pour, finalement, « redéfinir de façon graduelle mais irréversible, la nature même de la gouvernance » (Stone Sweet, 1999, p. 86).

Pour Ran Hirschl aussi, la « judiciarisation du politique [représente] un des phénomènes les plus significatifs de la gouvernance contemporaine » (Hirschl, 2002, p. 193). Mais pour Alec Stone Sweet, c'est l'établissement de Constitutions renforcées en Europe qui serait la principale illustration des profondes transformations de ce régime de gouvernance (Stone Sweet, 2000). De plus, ce ne serait pas seulement le niveau national qui serait concerné mais également le niveau supra-national. Ainsi, l'institution de la Cour Européenne aurait progressivement transformé la nature d’une gouvernance européenne. Celle-ci serait caractérisée, selon Alec Stone Sweet, par le fait que, dans le contexte actuel d’une politique 
européenne à multiple dimension, la souveraineté du Législatif et la primauté des Exécutifs nationaux seraient remis en cause (Stone Sweet, 2000 ; 2004).

Si nous rappelons ces perspectives d'analyse à l'issue de notre propos, c'est qu'elles illustrent à l'évidence la véritable nature des questions que soulève le phénomène de judiciarisation et les enjeux qu'il porte. Ce qui constitue pour nous un fait relevant d'une analyse de sociologie politique du droit, c’est que, quels que soient l'ampleur et les formes de sa réalité, ce phénomène de judiciarisation est posé, par des auteurs qui en sont parfois partie prenante et par des acteurs sociaux (professionnels du droit, personnel politique, mouvements sociaux, etc.) comme un phénomène d'importance ayant à voir avec la structuration du politique. L'affirmation d'un rôle croissant des cours suprêmes et, plus généralement des juridictions, dans la réalisation des politiques publiques, dans la gestion des droits fondamentaux, dans la régulation du politique, la thèse avancée d’une inscription de la justice dans des stratégies politiques, que ce soient celles des élites ou celles des mouvements sociaux, la conviction assumée que la supranationalisation accroît le rôle de la justice..., tout ceci sonne comme un rappel de la fonction stratégique de la légalité dans la constitution du politique. L’intérêt porté au phénomène de judiciarisation, la mobilisation exceptionnelle d'analyses et de réflexions dont il fait l'objet, ne seraient alors qu'une des manifestations suscitées par la prise de conscience d'un changement en cours du régime de la légalité (supranationalisation dans la production des normes juridiques et dans leur application, prééminence de plus en plus affirmée et quels que soient les systèmes de droit - droit romano-germanique et common law d’un droit-ressource par rapport à un droit référence, etc....). Ce changement du régime de légalité serait associé et participerait des transformations du politique (passage du gouvernement à la gouvernance, affaiblissement du modèle top down de régulation politique, bouleversement des territoires de la puissance publique liés à l'Etat nation, etc.) pour signifier 
notamment la remise en cause de l'Etat moderne ${ }^{7}$ et l'avènement d'un modèle de domination légitime dont la judiciarisation, réalisée ou représentée, serait alors un des attributs.

\section{$<$ BIBLIOGRAPHIE $>$}

Alter K. J. (1994), « Judicial Politics in the European Community. European Integration and the Pathbreaking Cassis de Dijon Decision”, Comparative Political Studies, Vol. 26, $\mathrm{n}^{\circ} 4$, January, p. 535-561.

Alter K. J. (1996), « The European Court's Political Power », West European Politics, Vol. 19, n 3 , July, p. 458-487.

Alter K. J. (1998), "Who are the "Masters of Treaty" ? : European Governments and the European Court of Justice”, International Organization, 52, 1, Winter, p. 121-147.

Alter K. J. (2006), "Private Litigants and the New International Courts", Comparative Political Studies, Vol. 39, n 1, p. 22-49.

Andersen E. A. (2005), Out of the Closets \& into the Courts. Legal Opportunity Structure and Gay Rights Litigation, The University of Michigan Press.

Auerbach J. S. (1983), Justice without Law ? Resolving Disputes without Lawyers, Oxford, Oxford University Press.

Cannon M. W. et O'Brien D. M. (Eds), Views from the Bench. The Judiciary and Constitutional Politics, Chatham, New Jersey, Chatham House Publishers Inc.

Canon B.C. and Johnson C.A. (1998), Judicial Policies : Implementation and Impact, Washington DC, CQ Press, $2^{\text {nd }}$ ed.

Champagne A. et Haydel J. (Eds) (1993), Judicial Reform in the States, Lanham, MD, University Press of America.

Chevallier J. (2004), L’Etat post-moderne, Paris, LGDJ, coll. « Droit et Société », série « Politique ».

Cichowski R. A. (2004), «Women's Rights, the European Court, and Supranational Constitutionnalism », Law \& Society, Vol. 38, n³, p. 489-512.

Cichowski R. A. (2006a), «Introduction. Courts, Democracy and Governance », Comparative Political Studies, Vol. 39, n 1, February, p. 3-21.

Cichowski R. A. (2006b), "Courts, Rights and Democratic Participation”, Comparative Political Studies, Vol. 39, n 1, February, 50-75.

Commaille J. (1989), «Les tribunaux de famille sous la Révolution. Recours à l'histoire comme contribution à une sociologie de la Justice et des relations privé-public », in R. Badinter (dir.), Une autre Justice. La Justice et la Révolution Française, Fayard, 1989, p. 205-223..

Commaille J. (2000), Territoires de justice. Une sociologie politique de la carte judiciaire, Paris, PUF.

Coman R. et De Waele J.-M. (2007), Judicial Reforms in Central and Eastern European Countries, Baden-Baden, Vanden Broele.

Conant L. (2006), "Individuals, Courts, and the Development of European Social Rights”, Comparative Political Studies, Vol.39, n 1, February, p. 76-100.

Dahl R., (1957), "The Supreme Court as a National Policymaker”, Journal of Public Law, vol.6, p. 279-295.

\footnotetext{
${ }^{7}$ Notons ici que Patrice Duran avait déjà justement associé la question du droit, ses métamorphoses nécessaires, à celle de la «remise en question de l'Etat comme élément central de la régulation des sociétés modernes » (Duran, 1993 ; voir également Chevallier, 2004).
} 
Dor G. et Hofnung M. (2006), « Litigation as Political Participation », Israel Studies, Vol. 11, $\mathrm{n}^{\circ}$ 2, p. 131-157.

Duran P. (1993), “Piloter l'action publique, avec ou sans le droit ?” Politiques et Management Public, Vol. 11, $\mathrm{n}^{\circ}$ 4, décembre, p. 1-45.

Edelman M., (1994), “The Judicialization of Politics in Israel”, International Political Science Review, vol. 15, n², p. 177-186.

Epp C. R. (1998), The Rights Revolution. Lawyers, Activists, and Supreme Courts in Comparative Perspective, Chicago, London, The University of Chicago Press.

Epstein L., Knight J., Martin A.D., (2001), "The Supreme Court as a Strategic National Policymaker”, Emory Law Journal, 50, p.583-611.

Epstein L., Knight J. et Shvetsova O. (2001), "The Role of Constitutional Courts in the Establishment and Maintenance of Democratic Systems of Government“, Law \& Society Review, Vol. 35, n 1, p. 117-163.

Epstein L. et Knight J. (2004), “Courts and Judges”, The Blackwell Companion to Law and Society, Malden (Mass.), Oxford, Blackwell Publishing Ltd, p. 170-194.

Malcolm M. Feeley et Rubin E. L. (1998), Judicial Policy Making and the Modern State. How the Courts Reformed America's Prisons, Cambridge, New York.

Ferejohn J., (2002), “Judicialization Politics, Politicizing Law", Law and Contemporary Problems, 65, 3, p. 41-68.

Fournier B. et Woehrling J. (2000), «Présentation du numéro Judiciarisation et pouvoir politique », Politique et Sociétés, Vol. 19, n²-3, p. 3-7.

Galanter M. (1974), "Why the "Haves" Come Out Ahead. Speculations on the Limits of Legal Change”, Law \& Society Review, n 9, p. 95-160.

Garcia Villegas M. (2004), “On Pierre Bourdieu’s Legal Thought”, Droit et Société, $n^{\circ}$ 56-57, p. 57-71.

George T. E. et Epstein L. (1992), « On the Nature of Supreme Court Decision Making », The American Political Science Review, Vol. 86, n² 2, June, p. 323-337.

Gillman H. (2001), "What's Law Got to Do with It ? Judicial Behavorialists Test the "Legal Model” of Judicial Decision Making”, Law \& Social Inquiry, Vol. 26, n² 2, Spring, p. 465504.

Gillman H. (2002), "How Political Parties Can Use the Courts to Advance Their Agendas : Federal Courts in the United States, (1875-1891)", American Political Science Review, Vol. 96, $\mathrm{n}^{\circ}$ 3, September, p. 511-524.

Ginsburg, T., (2003), Judicial Review in New Democracies. Constitutional Courts in Asian Cases, Cambridge, New York: Cambridge University Press.

Glick H. R. (1983), Courts, Politics and Justice, New York, McGraw Hill Book Company.

Glick H. R. (1992), “Judicial Innovation and Policy Re-Invention : State Supreme Courts and the Right to Die”, The Western Political Quarterly, Vol. 45, n 1, March, p. 71-92.

Guarnieri C. et Pederzoli P. (2002), From Democracy to Juristocracy? The Power of Judges : A Comparative Study of Courts and Democracy, Oxford, New York, Oxford University Press

Halliday T. C., Karpik L. et Feeley M. M. (2007), Fighting for Political Freedom. Comparative Studies of the Legal Complex and Political Liberalism, Oxford, Portland, Hart Publishing, Onati International Series in Law and Society.

Haltom W. et McCann M. (2004), Distorting the Law. Politics, Media, and the Litigation Crisis, Chicago, London, The University of Chicago Press.

Hirschl R. (2001), "The Political Origins of Judicial Empowerment through Constitutionalization. Lessons from Israël's Constitutional Revolution”, Comparative Politics, April, p. 315-335. 
Hirschl R. (2002), "Resituating the Judicialization of Politics : Bush v. Gore as a Global Trend”, Canadian Journal of Law and Jurisprudence, Vol. XV, n², July, p. 191-218.

Hirschl R. (2004), Towards Juristocracy. The Origins and Consequences of the New Constitutionalism, Cambridge (Mass.); London, Harvard University Press.

Holmström B., (1994), “The Judicialization of Politics in Sweden”, International Political Science Review, vol. 15, n 2, pp. 153-164.

Horowitz D. L. (1977), The Courts and Social Policy, Washington, The Brookings Institution. Jacob H. et al (1996), Courts, Law \& Politics in Comparative Perspective, New Haven, London, Yale University Press.

Kelemen R. D., “Adversarial Legalism and European Governance”, Comparative Political Studies, Vol. 39, n 1, February, p. 101-127.

Knopff R. (1998), "Populism and the Politics of Rights : The Dual Attack on Representative Democracy”, Canadian Journal of Political Science, XXXI : 4, December, p. 683-705.

Koopmans T., (2003), Courts and Political Institutions: A Comparative View, Cambridge, Cambridge University Press.

Lefranc S. (2007), "La justice dans l'après-violence politique”, in J. Commaille et M. Kaluszynski (dir.), La fonction politique de la justice, Paris, La Découverte, p. 273-291. McCann, M., (1994). Rights at Work. Pay Equity Reform and the Politics of Legal Mobilization, Chicago, Chicago University Press.

McCann, M. W. (2004), "Law and Social Movements", The Blackwell Companion to Law and Society, Malden (Mass.), Oxford, Blackwell Publishing Ltd, p. 506-522.

McGowan C. (1986), “A Reply to Judicialization”, Duke Law Journal, n² 2, April, p. 217237.

Manfredi C. P. (2004), Feminist Activism in the Supreme Court. Legal Mobilization and the Women's Legal Education and Action Fund, Vancouver, Toronto, UBC Press.

Maveety N. et Grosskopf A. (2004), « « Constrained » Constitutional Courts as Conduits for Democratic Consolidation », Law \& Society Review, Vol. 38, n 3, p. 463-488.

McGuire K. T. et Caldeira G. A. (1993), «Lawyers, Organized Interests, and the Law of Obscenity : Agenda Setting in the Supreme Court”, American Political Science Review, Vol. 87, $n^{\circ}$ 3, September, p. 717-726.

McGuire K. T. et Palmer B. (1996), « Issues, Agendas, and Decision Making on the Supreme Court », American Political Science Review, Vol. 90, n 4, December, p. 853-865.

Mehta P. B. (2007), “The Rise of Judicial Sovereignty”, Journal of Democracy, Vol. 18, $\mathrm{n}^{\circ}$ 2, April, p. 70-83.

Mezey S. G. (1996), Children in Court. Public Policymaking and Federal Court Decisions, State University of New York Press.

Mizrahi S. et Meydani A. (2003), « Political Participation through the Judicial System : Exit, Voice and Quasi-Exit in Israeli Society », Israel Studies, vol. 8, n 2, pp. 118-138.

Morton F. L. et Allen A. (2001), "Feminists and the Courts : Measuring Success in Interest Group Litigation in Canada”, Canadian Journal of Political Science, XXXIV : 1, March, p. 55-84.

Morton F. L. (2002), Law \& Politics and Judicial Process in Canada, Calgary, University of Calgary Press, 3e éd.

O'Leary R. (1989), “The Impact of Federal Court Decisions on the Policies and Administration of the U.S. Environmental Protection Agency”, Administrative Law Review, 41, Fall, p. 549-574.

Olson S. M. (1990), "Interest Group Litigation in Federal District Court : Beyond the Political Disadvantage Theory”, Journal of Politics, Vol. 52, n 3, August, p. 854-882.

Parsons T. (1962), “The Law and Social Control”, in W. M. Evan (Ed.), Law and Sociology : Exploratory Essays, Glencoe, III, Free Press, 1962, p. 56-72. 
Reid T. V. (1988), "Judicial Policy-Making and Implementation : An Empirical Examination”, The Western Political Quarterly, Vol. 41, n³ 3, September, p. 509-527.

Reus-Smit C. (Ed.) (2004), The Politics of International Law, Cambridge, New York, Cambridge University Press.

Revue internationale de science politique / International Political Science Review (1994), The Judicialization of Politics, Vol ; 15, $\mathrm{n}^{\circ} 2$, April.

Riddell T. Q. (2004), “The Impact of Legal Mobilization and Judicial Decisions : The Case of Official Minority-Language Education Policy in Canada for Francophones Outside Quebec”,

Law \& Society Review, Vol. 38, n 3, p. 583-610.

Rothmayr C. et L’Espérance A. (2007), “Le recours à la justice par les patients, chercheurs et. groupes religieux dans le domaine de la biomedecine”, in J. Commaille et M. Kaluszynski

(dir.), La fonction politique de la justice, Paris, La Découverte, p. 191-209.

Rosenberg G. N. (1991), The Hollow Hope: Can Courts Bring about Social Change ?, Chicago, University of Chicago.

Roussel V., (2003), "La judiciarisation du politique, réalités et faux semblants », Mouvements, $\mathrm{n}^{\circ}$ 29, pp. 12-18.

Sarat A. et Scheingold S. A. (2006), Cause Lawyers and Social Movements, Stanford University Press.

Scheppele K.L. (2002), "Declarations of Independence : Judicial Reactions to Political Pressure” in S.B. Burbank and B. Friedman (Eds), Judicial Independence at the Crossroads. An Interdisciplinary Approach, Thousand Oaks, Sage Publications

Shapiro M., (1994), “Juridicalization of Politics in the United States”, International Political Science Review, Vol. 15, n², 1994, pp. 101-112.

Shapiro M. et Stone A; (1994), "The New Constitutional Politics of Europe”, Comparative Political Studies, 26:4, p. 397-420.

Shapiro M. and Stone Sweet A. (2002), On Law, Politics \& Judicialization, Oxford, New York, Oxford University Press.

Sieder R., Schjolden L. et Angell A. (Eds) (2005), The Judicialization of Politics in Latin America, New York, Palgrave MacMillan.

Slaughter A.-M. (2000), “Judicial Globalization”, Virginia Journal of International Law, Vol. 40, p. 1103-1124.

Slaughter A.-M. (2003), “A Global Community of Courts”, Harvard International Law Journal, Vol. 44, $\mathrm{n}^{\circ}$ 1, Winter, p. 191-219.

Smith L. A. (1985), “Judicialization : The Twilight of Administrative Law”, Duke Law Journal, p. 427-466.

Smith C. E. (1997), Courts, Politics and the Judicial Process, Chicago, Nelson-Hall Publishers, $2^{\text {nd }}$ ed.

Smith M. (2005), "Social Movements and Judicial Empowerment : Courts, Public Policy, and Lesbian and Gay Organizing in Canada”, Politics \& Society, Vol. 33, n² 2, June, p. 327-353.

Stone Sweet A.(1999), "Judicialization and the Construction of Governance”, Comparative Political Studies, Vol. 32, n² 2, April, p. 147-184.

Stone Sweet A. (2000), Governing with Judges. Constitutional Politics in Europe, Oxford, New York, Oxford University Press.

Stone Sweet A., Sandholtz W. et Fligstein N. (Eds.) (2001), The Institutionalization of Europe, Oxford, New York, Oxford University Press.

Sugarman D., "From Unimaginable to Possible: Spain, Pinochet and the Judicialization of Power”, Journal of Spanish Cultural Studies, Vol. 3, n 1, p. 107-124.

Sunkin M., (1994), "Judicialization of Politics in the United Kingdom”, International Political Science Review, Vol. 15, n², pp. 125-133. 
Tate C. N. et Vallinder T.(1995), The Global Expansion of Judicial Power, New York, New York University Press.

Tate C. N. et Vallinder T. (1994), The Judicialization of Politics, International Political Science Review, vol. $15, \mathrm{n}^{\circ} 2$.

Trochev A. (2004), "Less Democracy, More Courts : A Puzzle of Judicial Review in Russia”, Law \& Society Review, Vol. 38, n³, p. 513-548.

Uprimny Yepes R. (2007), "La justice au coeur du politique : potentialités et risques d'une judiciarisation en Colombie », in J. Commaille et M. Kaluszynski (dir.), La fonction politique de la justice, Paris, La Découverte, p. 229-250.

Vallinder T. (1994), "The Judicialization of Politics. A World-Wide Phenomenon: Introduction”, International Political Science Review, Vol. 15, n², p. 91-99.

Vanberg G. (1998), «Abstract Judicial Review, Legislative Bargaining, and Policy Compromise », Journal of Theoretical Politics, 10 (3), p. 299-326.

Vig N. J. et Kraft M. E. (Eds) (1997), Environnmental Policy in the 1990s. Reform on Reaction, Washington, CQ Press.

Volcansek M. L. (1992), “The European Court of Justice : Supranational Policy-Making”, West European Politics, Vol. 15, n 3, p. 109-121.

Volcansek M. L. (Ed.) (1997), The Judicialisation of Politics : Contemporary Trends in Research on European and Other Courts, Gainsville, University Press of Florida.

Waltman J., (1996), "The Global Expansion of Judicial Power”, The American Political Science Review (Book Review : Comparative Politics), 90, 3, p. 684-685.

Wright V. (1999), “The Fifth Republic: from the Droit de l'Etat to the Etat de droit ?”, West European Politics, 22/4, p; 92-119.

\section{Résumé}

Un travail d'inventaire, loin d'être exhaustif, entrepris sur la littérature internationale portant sur la «judiciarisation » constitue le support de la présente analyse. Après avoir rappelé les différentes définitions données de la «judiciarisation » et tenté de mieux cerner ce qui relève d'une réalité ou de représentations construites d'un accroissement du rôle de la justice par rapport au politique, des illustrations sont fournies dans le domaine des politiques publiques et, plus généralement, dans celui du fonctionnement démocratique. Il apparaît finalement que ce qui semble être le véritable enjeu d'une exceptionnelle mobilisation de la connaissance sur cet objet: c'est un changement de régime de légalité, d’une légalité qui ne serait plus seulement fondement du pouvoir mais instrument de pouvoir. Ce changement est associé et participe des transformations du politique pour signifier notamment l'avènement d'un modèle de domination légitime dont la « judiciarisation », réalisée ou représentée, deviendrait alors un des attributs.

\section{Mots-clefs}

Démocratie - Régime de légalité - Gouvernance - Judiciarisation - Juridicisation - Politiques publiques. 
Title of article : Fortunes and misfortunes of legality in contemporary societies. A political sociology of "judicialization"

Authors: Jacques Commaille and Laurence Dumoulin

\section{Summary}

This article takes stock of the international literature on the "judicialization", although it is hardly exhaustive. After recalling the different definitions of "judicialization" and attempting to discern better what is reality and what are representations constructed by the increased role of justice, compared with politics. Illustrations are provided in the domain of public policy and, more generally, in the domain of democratic functioning. It appears that what seems to be the true issue of an exceptional mobilization of knowledge on this object is a regime change of legality, a legality that would no longer be the foundation of power, but an instrument of power. This change is associated with and draws on policy transformations to signify in particular the accession of a model of legitimate domination of which the “judicialization”, realized or represented, would then become one of its attributes.

\section{Keywords}

Democracy - Governance - Judicialization - Juridicization - Public policy - Regime of legality 